\title{
THE INFLUENCE OF THERMAL EVOLUTION IN THE MAGNETIC PROTECTION OF TERRESTRIAL PLANETS
}

\author{
Jorge I. Zuluaga ${ }^{1}$, Sebastian Bustamante ${ }^{1}$, Pablo A. Cuartas ${ }^{1}$, and Jaime H. Hoyos ${ }^{2}$ \\ ${ }^{1}$ Instituto de Física-FCEN, Universidad de Antioquia, Calle 67 No. 53-108, Medellín, Colombia; jzuluaga@ fisica.udea.edu.co, \\ sbustama@pegasus.udea.edu.co,p.cuartas@fisica.udea.edu.co \\ ${ }^{2}$ Departamento de Ciencias Básicas, Universidad de Medellín, Carrera 87 No. 30-65, Medellín, Colombia; jhhoyos@udem.edu.co \\ Received 2012 March 20; accepted 2013 April 10; published 2013 May 21
}

\begin{abstract}
Magnetic protection of potentially habitable planets plays a central role in determining their actual habitability and/ or the chances of detecting atmospheric biosignatures. Here we develop a thermal evolution model of potentially habitable Earth-like planets and super-Earths (SEs). Using up-to-date dynamo-scaling laws, we predict the properties of core dynamo magnetic fields and study the influence of thermal evolution on their properties. The level of magnetic protection of tidally locked and unlocked planets is estimated by combining simplified models of the planetary magnetosphere and a phenomenological description of the stellar wind. Thermal evolution introduces a strong dependence of magnetic protection on planetary mass and rotation rate. Tidally locked terrestrial planets with an Earth-like composition would have early dayside magnetopause distances between 1.5 and $4.0 R_{p}$, larger than previously estimated. Unlocked planets with periods of rotation $\sim 1$ day are protected by magnetospheres extending between 3 and $8 R_{p}$. Our results are robust in comparison with variations in planetary bulk composition and uncertainties in other critical model parameters. For illustration purposes, the thermal evolution and magnetic protection of the potentially habitable SEs GL 581d, GJ 667Cc, and HD 40307g were also studied. Assuming an Earth-like composition, we found that the dynamos of these planets are already extinct or close to being shut down. While GL 581d is the best protected, the protection of HD 40307g cannot be reliably estimated. GJ 667Cc, even under optimistic conditions, seems to be severely exposed to the stellar wind, and, under the conditions of our model, has probably suffered massive atmospheric losses.
\end{abstract}

Key words: planetary systems - planets and satellites: magnetic fields - planets and satellites: physical evolution planet-star interactions

Online-only material: color figures

\section{INTRODUCTION}

The discovery of extrasolar habitable planets is one of the most ambitious challenges in exoplanetary research. At the time of writing, there are almost 861 confirmed exoplanets ${ }^{3}$ including 61 classified as Earth-like planets (EPs, $M \sim 1 M_{\oplus}$ ) and super-Earths (SEs, $M \sim 1-10 M_{\oplus}$; Valencia et al. 2006, hereafter VAL06). Among these low-mass planets, there are three confirmed SEs, GJ 667Cc (Bonfils et al. 2011), GL 581d (Udry et al. 2007; Mayor et al. 2009), and HD 40307g (Tuomi et al. 2012), and tens of Kepler candidates (Borucki et al. 2011; Batalha et al. 2012) that are close to or inside the habitable zone (HZ) of their host stars (see, e.g., Selsis et al. 2007; Pepe et al. 2011; Kaltenegger et al. 2011). If we include the possibility that giant exoplanets could harbor habitable exomoons, the number of the potentially habitable planetary environments already discovered beyond the solar system could be raised to several tens (Underwood et al. 2003; Kaltenegger 2010). Moreover, the existence of a plethora of other terrestrial planets (TPs) and exomoons in the Galaxy is rapidly gaining evidence (Borucki et al. 2011; Catanzarite \& Shao 2011; Bonfils et al. 2011; Kipping et al. 2012), and the chances that a large number of potentially habitable extrasolar bodies could be discovered in the near future are very large.

The question of which properties a planetary environment need in order to allow the appearance, evolution, and diversification of life has been extensively studied (for recent reviews,

\footnotetext{
3 For updates, please refer to http://exoplanet.eu
}

see Lammer et al. 2009 and Kasting 2010). Two basic and complementary physical conditions must be fulfilled: the presence of an atmosphere and the existence of liquid water on the surface (Kasting et al. 1993). However, the fulfillment of these basic conditions depends on many complex and diverse endogenous and exogenous factors (for a comprehensive enumeration of these factors, see, e.g., Ward \& Brownlee 2000 or Lammer et al. 2010).

The existence and long-term stability of an intense planetary magnetic field (PMF) is one of these relevant factors (see, e.g., Grießmeier et al. 2010 and references therein). It has been shown that a strong enough PMF could protect the atmosphere of potentially habitable planets, especially its valuable content of water and other volatiles, against the erosive action of the stellar wind (Lammer et al. 2003, 2007; Khodachenko et al. 2007; Chaufray et al. 2007). Planetary magnetospheres also act as shields against the potentially harmful effects that the stellar high energy particles and galactic cosmic rays (CR) produce in the life forms evolving on the planetary surface (see, e.g., Grießmeier et al. 2005). Even in the case that life could arise and evolve on unmagnetized planets, the detection of atmospheric biosignatures would also be affected by a higher flux of high energy particles including $\mathrm{CR}$, especially if the planet is close to very active M-dwarfs (dM; Grenfell et al. 2007; Segura et al. 2010).

It has recently been predicted that most of the TPs in our Galaxy could be found around dM stars (Boss 2006; Mayor \& Udry 2008; Scalo et al. 2007; Rauer et al. 2011; Bonfils et al. 2011). Actually, $20 \%$ of the presently confirmed SEs belong 
to planetary systems around stars of this type. Planets in the HZ of low-mass stars $\left(M_{\star} \lesssim 0.6 M_{\odot}\right)$ would be tidally locked (Joshi et al. 1997; Heller et al. 2011), a condition that poses serious limitations to their potential habitability (see, e.g., Kite et al. 2011 and references therein). Tidally locked planets inside the $\mathrm{HZ}$ of dMs have periods in the range of 5-100 days, a condition that has commonly been associated with the almost complete lack of a protective magnetic field (Grießmeier et al. 2004). However, the relation between rotation and PMF properties, which is critical at assessing the magnetic protection of slowly rotating planets, is more complex than previously thought. In particular, a detailed knowledge of the thermal evolution of the planet is required to predict not only the intensity but also the regime (dipolar or multipolar) of the PMF for a given planetary mass and rotation rate (Zuluaga \& Cuartas 2012).

Although several authors have extensively studied the protection that intrinsic PMF would provide to extrasolar planets (Grießmeier et al. 2005; Khodachenko et al. 2007; Lammer et al. 2007; Grießmeier et al. 2009, 2010), all have disregarded the influence that thermal evolution has in the evolution of planetary magnetic properties. They have also used outdated dynamo scaling laws that have been recently revised (see Christensen 2010 and references therein). The role of rotation in determining the PMF properties that is critical in assessing the case of tidally locked planets has also been overlooked (Zuluaga \& Cuartas 2012).

We develop a comprehensive model for the evolution of the magnetic protection of potentially habitable TPs around GKM main-sequence stars. To achieve this goal, we integrate in a single framework a parameterized thermal evolution model based on the most recent advances in the field (Gaidos et al. 2010; Tachinami et al. 2011; Stamenković et al. 2012), up-todate dynamo scaling laws (Christensen 2010; Zuluaga \& Cuartas 2012), and phenomenological models for the evolution of the stellar wind and planet-star magnetic interaction (Grießmeier et al. 2010). Our model is aimed at (1) understanding the influence of thermal evolution in the magnetic protection of TPs, (2) assessing the role of low rotation periods in the evolution of the magnetic protection of tidally locked habitable planets, (3) placing more realistic constraints on the magnetic properties of potentially habitable TPs suitable for future studies of atmospheric mass loss or the CR effect on the atmospheric chemistry or on life itself, and (4) estimating by the first time the magnetic properties of SEs already discovered in the $\mathrm{HZ}$ of their host stars.

Our work is a step forward in the understanding of planetary magnetic protection because it combines in a single model the evolution of the magnetic properties of the planet and hence its dependence on planetary mass and composition and the role of the planet-star interaction into determining the resulting level of magnetic protection. Previous models of the former (thermal evolution and intrinsic magnetic properties) did not consider the interaction of the PMF with the evolving stellar wind which is finally the factor that determines the actual level of planetary magnetic protection. On the other hand, previous attempts to study the planet-star interaction overlooked the nontrivial dependence of intrinsic magnetic properties on planetary thermal evolution and hence on planetary mass and rotation rate. Additionally, and for the first time, we are attempting here to calculate the magnetic properties of the potentially habitable SEs already discovered, GJ 667Cc, Gl 581d, and HD 40307g.

This paper is organized as follows. Section 2 is aimed at introducing the properties of planetary magnetospheres we should estimate in order to evaluate the level of magnetic protection of a potentially habitable TP. Once those properties are expressed in terms of two basic physical quantities, the planetary magnetic dipole moment and the pressure of the stellar wind, we proceed to describe how those quantities can be estimated by modeling the thermal evolution of the planet (Section 3), scaling the dynamo properties from the planetary thermal and rotational properties (Section 4), and modeling the interaction between the star and the planet (Section 5). In Section 6, we apply our model to evaluate the level of magnetic protection of hypothetical potentially habitable TPs as well as the habitable SEs already discovered. We also present the results of a numerical analysis aimed at evaluating the sensitivity of our model to uncertainties in the composition of the planet and to other critical parameters of the model. In Section 7, we discuss the limitations of our model, present an example of the way in which our results could be applied to estimate the mass-loss rate from already and yet to be discovered potentially habitable TPs, and discuss the observational prospects to validate or improve the model. Finally, a summary and several conclusions drawn from this research are presented in Section 8.

\section{CRITICAL PROPERTIES OF AN EVOLVING MAGNETOSPHERE}

The interaction between the PMF, the interplanetary magnetic field (IMF), and the stellar wind creates a magnetic cavity around the planet known as the magnetosphere. Although magnetospheres are very complex systems, their global properties are continuous functions of only two physical variables (Siscoe \& Christopher 1975): the magnetic dipole moment of the planet, $\mathcal{M}$, and the dynamic pressure of the stellar wind, $P_{\text {sw }}$. Dipole moment is defined in the multipolar expansion of the magnetic field strength:

$$
B_{p}(r)=\frac{\mu_{0} \mathcal{M}}{4 \pi r^{3}}+\mathcal{O}\left(\frac{1}{r^{4}}\right),
$$

where $B_{p}(r)$ is the angular-averaged PMF strength measured at a distance $r$ from the planet center and $\mu_{0}=4 \pi \times 10^{-7} \mathrm{H} \mathrm{m}^{-1}$ is the vacuum permeability. In the rest of the paper, we will drop off the higher order terms in $1 / r$ (multipolar terms) and focus on the dipolar component of the field $B_{p}^{\text {dip }}$ which is explicitly given by the first term of the right side in Equation (1).

The dynamic pressure of the stellar wind is given by

$$
P_{\mathrm{sw}}=m n v_{\mathrm{eff}}^{2}+2 n k_{B} T
$$

where $m$ and $n$ are the typical mass of a wind particle (mostly protons) and its number density, respectively. Here, $v_{\text {eff }}=$ $\left(v_{\mathrm{sw}}^{2}+v_{p}^{2}\right)^{1 / 2}$ is the effective velocity of the stellar wind as measured in the reference frame of the planet whose orbital velocity is $v_{p} . T$ is the local temperature of the wind plasma and $k_{B}=1.38 \times 10^{-23} \mathrm{~J} \mathrm{~K}^{-1}$ is the Boltzmann constant.

There are three basic properties of planetary magnetospheres we are interested in: (1) the maximum magnetopause field intensity $B_{\mathrm{mp}}$, which is a proxy of the flux of high energy particles entering into the magnetospheric cavity; (2) the standoff or stagnation radius, $R_{S}$, a measure of the size of the dayside magnetosphere; and (3) the area of the polar cap $A_{\mathrm{pc}}$ that measures the total area of the planetary atmosphere exposed to open field lines through which particles can escape to interplanetary space. The value of these quantities provides information about 
the level of exposure that a habitable planet has to the erosive effects of stellar wind and the potentially harmful effects of the CR.

The maximum value of the magnetopause field intensity $B_{\mathrm{mp}}$ is estimated from the balance between the magnetic pressure $P_{\mathrm{mp}}=B_{\mathrm{mp}}^{2} /\left(2 \mu_{0}\right)$ and the dynamic stellar wind pressure $P_{\mathrm{sw}}$ (Equation (2)),

$$
B_{\mathrm{mp}}=\left(2 \mu_{0}\right)^{1 / 2} P_{\mathrm{sw}}^{1 / 2} .
$$

Here, we are assuming that the pressure exerted by the plasma inside the magnetospheric cavity is negligible (see the discussion below).

Although magnetopause fields arise from very complex processes (Chapman-Ferraro and other complex currents at the magnetosphere boundary), in simplified models $B_{\mathrm{mp}}$ is assumed to be proportional to the PMF intensity $B_{p}$ as measured at the substellar point $r=R_{S}$ (Mead 1964; Voigt 1995),

$$
B_{\mathrm{mp}}=2 f_{0} B_{p}\left(r=R_{S}\right) \approx\left(\frac{f_{0} \mu_{0}}{2 \pi}\right) \sqrt{2} \mathcal{M} R_{S}^{-3} .
$$

$f_{0}$ is a numerical enhancement factor of the order of one that can be estimated numerically. We are assuming here that the dipolar component of the intrinsic field (the first term on the right-hand side of Equation (1)) dominates at magnetopause distances even in slightly dipolar PMF.

Combining Equations (3) and (4) we estimate the standoff distance:

$$
R_{S}=\left(\frac{\mu_{0} f_{0}^{2}}{8 \pi^{2}}\right)^{1 / 6} \mathcal{M}^{1 / 3} P_{\mathrm{sw}}^{-1 / 6}
$$

which can be expressed in terms of the present dipole moment of the Earth $\mathcal{M}_{\oplus}=7.768 \times 10^{22} \mathrm{~A} \mathrm{~m}^{2}$ and the average dynamic pressure of the solar wind as measured at the orbit of our planet $P_{\text {sw } \odot}=2.24 \times 10^{-9} \mathrm{~Pa}($ Stacey 1992; Grießmeier et al. 2005):

$$
\frac{R_{S}}{R_{\oplus}}=9.75\left(\frac{\mathcal{M}}{\mathcal{M}_{\oplus}}\right)^{1 / 3}\left(\frac{P_{\mathrm{sw}}}{P_{\mathrm{sw} \odot}}\right)^{-1 / 6} .
$$

It is important to stress that the value of $R_{S}$ estimated with Equation (5) assumes a negligible value of the plasma pressure inside the magnetospheric cavity. This approximation is valid if at least one of these conditions is fulfilled: (1) the PMF is very intense, (2) the dynamic pressure of the stellar wind is small, or (3) the planetary atmosphere is not too bloated by the XUV radiation. In the case when any of these conditions are fulfilled, we will refer to $R_{S}$ as given by Equation (5) as the magnetic standoff distance which is an underestimation of the actual size of the magnetosphere.

Last, but not least, we are interested in evaluating the area of the polar cap. This is the region in the magnetosphere where magnetic field lines could be open into the interplanetary space or to the magnetotail region. Siscoe \& Chen (1975) have shown that the area of the polar cap $A_{\mathrm{pc}}$ scales with dipole moment and dynamic pressure as

$$
\frac{A_{\mathrm{pc}}}{4 \pi R_{p}^{2}}=4.63 \%\left(\frac{\mathcal{M}}{\mathcal{M}_{\oplus}}\right)^{-1 / 3}\left(\frac{P_{\mathrm{sw}}}{P_{\mathrm{sw} \odot}}\right)^{1 / 6}
$$

Here, we have normalized the polar cap area with the total area of the atmosphere $4 \pi R_{p}^{2}$, and assumed the atmosphere has a scale height much smaller than planetary radius $R_{p}$.
In order to model the evolution of these three key magnetosphere properties we need to estimate the surface dipolar component of the PMF $B_{p}^{\mathrm{dip}}\left(R_{p}\right)$ (from which we can obtain the dipole moment $\mathcal{M}$ ), the average number density $n$, velocity $v_{\text {eff }}$, and temperature $T$ of the stellar wind (which are required to predict the dynamic pressure $P_{\mathrm{sw}}$ ). These quantities depend in general on time and also on different planetary and stellar properties. In the following sections, we describe our model for the calculation of the evolving values of these fundamental quantities.

\section{THERMAL AND DYNAMO EVOLUTION}

We assume here that the main source of a global PMF in TPs is the action of a dynamo powered by convection in a liquid metallic core (Stevenson 1983, 2003). This assumption is reasonable since the Moon and all rocky planets in the solar system, regardless of their different origins and compositions, seem to presently have, or to previously had, an iron core dynamo (see, e.g., Stevenson 2010). Other potential sources of PMFs, such as body currents induced by the stellar magnetic field or dynamo action in a mantle of ice, water, or magma, are not considered here but left for future research.

The properties and evolution of a core dynamo will depend on the internal structure and thermal history of the planet. Thermal evolution of TPs, specially the Earth itself, has been studied for decades (for a recent review, see Nimmo 2009). A diversity of thermal evolution models for planets larger than the Earth have recently appeared in literature (Papuc \& Davies 2008; Gaidos et al. 2010; Tachinami et al. 2011; Driscoll \& Olson 2011; Stamenković et al. 2011). But the lack of observational evidence against which we can compare the predictions of these models has left too much room for uncertainties, especially regarding mantle rheology, core composition, and thermodynamic properties. Albeit these fundamental limitations, a global picture of the thermal history of SEs has started to arise. Here, we follow the lines of Labrosse (2003) and Gaidos et al. (2010) developing a parameterized thermal evolution model which combines a simplified model of the interior structure and a parameterized description of the core and mantle rheology.

Our model includes several distinctive characteristics in comparison to previous ones. The most important one is an upto-date treatment of mantle rheology. For that purpose, we use two different formulae to compute the viscosity of the upper and lower mantles. By lower mantle, we understand here the region of the mantle close to the core-mantle boundary (CMB). This is the hottest part of the mantle. The upper mantle is the outer cold part of this layer. It is customary to describe both regions with the same rheology albeit their very different mineralogical compositions. Additionally, thermal and density profiles in the mantle follow the same prescription as in the core. We also use a different ansatz to assign initial values to lower mantle temperature and to the temperature contrast across the CMB, two of the most uncertain quantities in thermal evolution models. Using our ansatz, we avoid assigning arbitrary initial values to these critical parameters but more importantly we are able to find a unified method to set the value of these temperatures in planets with very different masses. It is also important to note that in other models these temperatures were set by hand or were treated as free parameters in the model.

Four key properties should be predicted by any thermal evolution model in order to calculate the magnetic properties of a planet: (1) the total available convective power $Q_{\text {conv }}$, providing the energy required for magnetic field amplification through 
dynamo action; (2) the radius of the solid inner core $R_{\mathrm{ic}}$ and from there the height $D \approx R_{c}-R_{\text {ic }}$ of the convecting shell where the dynamo action takes place $\left(R_{c}\right.$ is the radius of the core); (3) the time of inner core formation $t_{\text {ic }}$; and (4) the total dynamo lifetime $t_{\text {dyn }}$.

In order to calculate these quantities, we solve simply parameterized energy and entropy equations of balance describing the flux of heat and entropy in the planetary core and mantle. As stated before, our model is based on the interior structure model by VAL06 and in thermal evolution models previously developed by Schubert et al. (1979), Stevenson (1983), Nimmo \& Stevenson (2000), Labrosse et al. (2001), Labrosse (2003), Gubbins et al. (2003, 2004), Aubert et al. (2009), Gaidos et al. (2010), and Stamenković et al. (2011). For a detailed description of the fundamental physics behind the thermal evolution model developed here, please refer to these earlier studies.

\subsection{Interior Structure}

Our one-dimensional model for the interior assumes a planet made by two well-differentiated chemically and mineralogically homogeneous shells: a rocky mantle made out of olivine and perovskite and a core made by iron plus other light elements.

The mechanical conditions inside the planet (pressure $P$, density $\rho$, and gravitational field $g$ ) are computed by simultaneously solving the continuity, Adams-Williamson, and hydrostatic equilibrium equations (Equations (1)-(4) in VAL06). For all planetary masses, we assume boundary conditions, $\rho\left(r=R_{p}\right)=4000 \mathrm{~kg} \mathrm{~m}^{-3}$ and $P\left(r=R_{p}\right)=0 \mathrm{~Pa}$. For each planetary mass and core mass fraction $\mathrm{CMF}=M_{\text {core }} / M_{p}$, we use an RK4 integrator and a shooting method to consistently compute the core $R_{c}$ and planetary radius $R_{p}$.

For the sake of simplicity, we do not include in the interior model the two- or even three-layered structure of the mantle. Instead, we assume a mantle completely made of perovskite-postperovskite (ppv). This is the reason why we take $\rho\left(r=R_{p}\right)=4000 \mathrm{~kg} \mathrm{~m}^{-3}$ instead of the more realistic value of $3000 \mathrm{~kg} \mathrm{~m}^{-3}$. With a single layer and a realistic surface density, our model is able to reproduce the present interior properties of the Earth.

In all cases, we use the Vinet equation of state instead of the commonly used third-order Birch-Murnaghan equation (BM3). It is well known that the BM3 follows from a finite strain expansion and does not accurately predict the properties of the material for the typical pressures found in SEs, i.e., 100-1000 GPa for $M_{p}=1-10 M_{\oplus}$ (VAL06; Tachinami et al. 2011). We have ignored thermal corrections to the adiabatic compressibility, i.e., $K_{S}(\rho, T) \approx K_{S}(\rho, 300 \mathrm{~K})+\Delta K_{S}(T)$ (VAL06). This assumption allows us to decouple at runtime the CPU intensive calculation of the thermal profile from the mechanical structure at each time step in the thermal evolution integration.

Although we have ignored the "first-order" effect of the temperature in the mechanical structure, we have taken into account "second-order" effects produced by phase transitions inside the mantle and core. Using the initial temperature profile inside the mantle, we calculate the radius of transition from olivine to perovskite (neglecting the effect of an intermediate layer of wadsleyite). For that purpose, we use the reduced pressure function $\Pi$ (Christensen 1985; Weinstein 1992; Valencia et al. 2007). For simplicity, the position of the transition layer is assumed to be constant during the whole thermal evolution of the planet. We have verified that this assumption does not significantly change the mechanical properties inside the planet, at least not at a level affecting the thermal evolution itself.
Inside the core, we continuously update the radius of the transition from solid to liquid iron (see below). For that purpose, we use the thermal profile computed at the previous time step. To avoid a continuous update of the mechanical structure, we assume in all the cases that during the transition from solid to liquid the density of iron changes by a constant factor $\Delta \rho=\left(\rho_{s}-\rho_{l}\right) / \rho_{l}$. Here, the reference density of the solid $\rho_{s}$ (when applied) is computed using the Vinet equation evaluated at a point in the very center of the planet.

Table 1 enumerates the relevant physical parameters of the interior structure and thermal evolution model.

\subsection{Core Thermal Evolution}

In order to compute the thermal evolution of the core, we solve the equations of energy and entropy balance (Labrosse et al. 2001; Nimmo 2009):

$$
\begin{gathered}
Q_{c}=Q_{s}+f_{i}\left(Q_{g}+Q_{l}\right) \\
\Phi=E_{l}+f_{i}\left(E_{s}+E_{g}\right)-E_{k} .
\end{gathered}
$$

Here, $Q_{c}$ is the total heat flowing through the CMB and $\Phi$ is the total entropy dissipated in the core. $E_{S}$ and $Q_{s}$ are the entropy and heat released by the secular cooling, $E_{g}$ and $Q_{g}$ are the contribution to entropy and heat due to the redistribution of gravitational potential when light elements are released at the liquid-solid interface (buoyant energy), $E_{l}$ and $Q_{l}$ are the entropy and heat released by the phase transition (latent heat), and $E_{k}$ is a term accounting for the sink of entropy due to the conduction of heat along the core. We have avoided the terms coming from radioactive and pressure heating because their contribution is negligible at the typical conditions inside SEs (Nimmo 2009). As long as the buoyant and latent entropy and heat are only present when a solid inner core exists, we have introduced a boolean variable $f_{i}$ that turns on these terms when the condition for the solidification of the inner core arises.

The terms in the energy and entropy balance are a function of the time derivative of the temperature profile $\partial T(r, t) / \partial t$ (for detailed expressions of these terms, see Table 1 in Nimmo 2009). As an example, the secular heat and entropy are given by

$$
\begin{aligned}
Q_{s} & =-\int \rho c_{p} \frac{\partial T(r, t)}{\partial t} d V, \\
E_{s} & =-\int \rho c_{p}\left[\frac{1}{T_{c}(t)}-\frac{1}{T(r, t)}\right] \frac{\partial T(r, t)}{\partial t} d V .
\end{aligned}
$$

Here, $c_{p}$ is the specific heat of the core alloy and $T_{c}$ is the temperature at the $\mathrm{CMB}$. If we assume that the temperature profile of the core does not change during the thermal evolution, we can write temperature as $T(r, t)=f_{c}(r) T_{c}(t)$. Here, $f_{c}(r)$ is the core temperature radial profile that we will assume adiabatic (see below). It should be noted again that $T_{c}(t)=T\left(r=R_{c}, t\right)$.

With this assumption the energy balance equation (7) can be written as a first-order differential equation on $T_{c}$ :

$$
Q_{c}=M_{c}\left[C_{s}+f_{i}\left(C_{g}+C_{l}\right)\right] \frac{d T_{c}}{d t},
$$

where $M_{c}$ is the total mass of the core and $C_{s}, C_{g}$, and $C_{l}$ are core bulk heat capacities which can be expressed as volumetric integrals of the radial profile $f_{c}(r)$. In this equation, the total heat $Q_{c}$ is intrinsically a function of $T_{c}$ and should be computed independently (see below). 
Table 1

RTEM Parameters

\begin{tabular}{|c|c|c|c|}
\hline Parameter & Definition & Value & Ref. \\
\hline \multicolumn{4}{|c|}{ Bulk } \\
\hline $\mathrm{CMF}$ & Core mass fraction & 0.325 & $\cdots$ \\
\hline$T_{s}$ & Surface temperature & $290 \mathrm{~K}$ & $\cdots$ \\
\hline$\underline{P_{s}}$ & Surface pressure & 0 bar & $\cdots$ \\
\hline \multicolumn{4}{|c|}{ Inner core } \\
\hline$\cdots$ & Material & $\mathrm{Fe}$ & A \\
\hline$\rho_{0}, K_{0}, K_{0}^{\prime}, \gamma_{0}, q, \theta_{0}$ & Equation of state parameters & $8300 \mathrm{~kg} \mathrm{~m}^{-3}, 160.2 \mathrm{GPa}, 5.82,1.36,0.91,998 \mathrm{~K}$ & A \\
\hline$k_{c}$ & Thermal conductivity & $40 \mathrm{~W} \mathrm{~m}^{-1} \mathrm{~K}^{-1}$ & $\mathrm{~B}$ \\
\hline$\Delta S$ & Entropy of fusion & $118 \mathrm{~J} \mathrm{~kg}^{-1} \mathrm{~K}^{-1}$ & $\mathrm{C}$ \\
\hline \multicolumn{4}{|c|}{ Outer core } \\
\hline . & Material & $\mathrm{Fe}_{(0,8)} \mathrm{FeS}_{(0,2)}$ & A \\
\hline$\rho_{0}, K_{0}, K_{0}^{\prime}, \gamma_{0}, q, \theta_{0}$ & Equation of state parameters & $7171 \mathrm{~kg} \mathrm{~m}^{-3}, 150.2 \mathrm{GPa}, 5.675,1.36,0.91,998 \mathrm{~K}$ & A \\
\hline$\alpha$ & Thermal expansivity & $1.4 \times 10^{-6} \mathrm{~K}^{-1}$ & $\mathrm{D}$ \\
\hline$c_{p}$ & Specific heat & $850 \mathrm{~J} \mathrm{~kg}^{-1} \mathrm{~K}^{-1}$ & $\mathrm{C}$ \\
\hline$k_{c}$ & Thermal conductivity & $40 \mathrm{~W} \mathrm{~m}^{-1} \mathrm{~K}^{-1}$ & $\mathrm{~B}$ \\
\hline$\kappa_{c}$ & Thermal diffusivity & $6.5 \times 10^{-6} \mathrm{~m}^{2} \mathrm{~s}^{-1}$ & $\mathrm{E}$ \\
\hline$\Delta S$ & Entropy of fusion & $118 \mathrm{~J} \mathrm{~kg}^{-1} \mathrm{~K}^{-1}$ & $\mathrm{C}$ \\
\hline$\epsilon_{\mathrm{adb}}$ & Adiabatic factor for $T_{c}(t=0)$ & 0.7 & $\cdots$ \\
\hline$\xi_{c}$ & Weight of $T_{c}$ in core viscosity & 0.4 & $\cdots$ \\
\hline \multicolumn{4}{|c|}{ Lower mantle } \\
\hline$\cdots$ & Material & $\mathrm{pv}+\mathrm{fmw}$ & A \\
\hline$\rho_{0}, K_{0}, K_{0}^{\prime}, \gamma_{0}, q, \theta_{0}$ & Equation of state parameters & $4152 \mathrm{~kg} \mathrm{~m}^{-3}, 223.6 \mathrm{GPa}, 4.274,1.48,1.4,1070 \mathrm{~K}$ & A \\
\hline$d, m, A, b, D_{0}, m_{\mathrm{mol}}$ & Viscosity parameters & $1 \times 10^{-3} \mathrm{~m}, 2,13.3,12.33,2.7 \times 10^{-10} \mathrm{~m}^{2} \mathrm{~s}^{-1}, 0.10039 \mathrm{~kg} \mathrm{~mol}^{-1}$ & $\mathrm{~F}$ \\
\hline$\alpha$ & Thermal expansivity & $2.4 \times 10^{-6} \mathrm{~K}^{-1}$ & $\mathrm{D}$ \\
\hline$c_{p}$ & Specific heat & $1250 \mathrm{~J} \mathrm{~kg}^{-1} \mathrm{~K}^{-1}$ & $\mathrm{C}$ \\
\hline$k_{m}$ & Thermal conductivity & $6 \mathrm{~W} \mathrm{~m}^{-1} \mathrm{~K}^{-1}$ & $\mathrm{C}$ \\
\hline$\kappa_{m}$ & Thermal diffusivity & $7.5 \times 10^{-7} \mathrm{~m}^{2} \mathrm{~s}^{-1}$ & $\mathrm{E}$ \\
\hline$\Delta S$ & Entropy of fusion & $130 \mathrm{~J} \mathrm{~kg}^{-1} \mathrm{~K}^{-1}$ & $\mathrm{C}$ \\
\hline \multicolumn{4}{|c|}{ Upper mantle } \\
\hline$\cdots$ & Material & Olivine & A \\
\hline$\rho_{0}, K_{0}, K_{0}^{\prime}, \gamma_{0}, q, \theta_{0}$ & Equation of state parameters & $3347 \mathrm{~kg} \mathrm{~m}^{-3}, 126.8 \mathrm{GPa}, 4.274,0.99,2.1,809 \mathrm{~K}$ & A \\
\hline$B, n, E^{*}, \dot{\epsilon}$ & Viscosity parameters & $3.5 \times 10^{-15} \mathrm{~Pa}^{-\mathrm{n}} \mathrm{s}^{-1}, 3,430 \times 10^{3} \mathrm{j} \mathrm{mol}^{-1}, 1 \times 10^{-15} \mathrm{~s}^{-1}$ & $\mathrm{D}$ \\
\hline$V^{*}$ & Activation volume & $2.5 \times 10^{-6} \mathrm{~m}^{3} \mathrm{~mol}^{-1}$ & $\mathrm{~F}$ \\
\hline$\alpha$ & Thermal expansivity & $3.6 \times 10^{-6} \mathrm{~K}^{-1}$ & $\mathrm{D}$ \\
\hline$c_{p}$ & Specific heat & $1250 \mathrm{~J} \mathrm{~kg}^{-1} \mathrm{~K}^{-1}$ & $\mathrm{C}$ \\
\hline$k_{m}$ & Thermal conductivity & $6 \mathrm{~W} \mathrm{~m}^{-1} \mathrm{~K}^{-1}$ & $\mathrm{C}$ \\
\hline$\kappa_{m}$ & Thermal diffusivity & $7.5 \times 10^{-7} \mathrm{~m}^{2} \mathrm{~s}^{-1}$ & $\mathrm{E}$ \\
\hline$\theta$ & Potential temperature & $1700 \mathrm{~K}$ & $\mathrm{~F}$ \\
\hline$\chi_{r}$ & Radioactive heat correction & 1.253 & $\cdots$ \\
\hline
\end{tabular}

Note. Sources: (A) VAL06, (B) Nimmo (2009), (C) Gaidos et al. (2010), (D) Tachinami et al. (2011), (E) Ricard (2009), (F) Stamenković et al. (2011).

Using a simple exponential fit for the core density, as proposed by Labrosse et al. (2001), the adiabatic temperature profile can be approximated as (Labrosse 2003)

$$
f_{c}(r)=\exp \left(\frac{R_{c}^{2}-r^{2}}{D_{c}^{2}}\right)
$$

where $D_{c}=\sqrt{3 c_{p} / 2 \pi \alpha \rho_{c} G}$ is the scale height of temperature, $\alpha$ is the isothermal expansivity (assumed for simplicity constant along the core), and $\rho_{c}$ is the density at core center. Using this fit, the bulk secular heat capacity $C_{s} \equiv Q_{s} /\left(M_{c} d T_{c} / d t\right)$ can be obtained from Equation (9):

$$
C_{s}=-4 \pi \int_{0}^{R_{c}} \rho(r) c_{p} \exp \left(\frac{R_{c}^{2}-r^{2}}{D_{c}^{2}}\right) r^{2} d r .
$$

Analogous expressions for $C_{g}$ and $C_{l}$ are obtained from the definition of $Q_{g}$ and $Q_{l}$ as given in Table 1 of Nimmo (2009).
The total heat released by the core $Q_{c}\left(T_{c}\right)$ is calculated here using the boundary layer theory (BLT; see, e.g., Stevenson 1983). Under this approximation $Q_{c}$ is given by (Ricard 2009)

$$
Q_{c}=4 \pi R_{c} k_{m} \Delta T_{\mathrm{CMB}} \mathrm{Nu}_{c},
$$

where $k_{m}$ is the thermal conductivity of the lower mantle, $\Delta T_{\mathrm{CMB}}=T_{c}-T_{l}$ is the temperature contrast across the CMB, $T_{l}$ is the lower mantle temperature, and $\mathrm{Nu}_{c} \approx\left(\mathrm{Ra}_{c} / \mathrm{Ra}_{*}\right)^{1 / 3}$ is the Nusselt number at the core (Schubert et al. 2001). The critical Rayleigh number $\mathrm{Ra}_{*}$ is a free parameter in our model (see Table 1).

The local Rayleigh number $\mathrm{Ra}_{c}$ at the CMB is calculated under the assumption of a boundary heated from below (Ricard 2009),

$$
\operatorname{Ra}_{c}=\frac{\rho g \alpha \Delta T_{\mathrm{CMB}}\left(R_{p}-R_{c}\right)^{3}}{\kappa_{c} \eta_{c}},
$$


where $g$ is the gravitational field and $\kappa_{c}$ the thermal diffusivity at the CMB. The value of the dynamic viscosity $\eta_{c}$, which is strongly dependent on temperature, could be suitably computed using the so-called film temperature (see Manga et al. 2001 and references therein). This temperature could be computed in general as a weighted average of the temperatures at the boundaries,

$$
T_{\eta c}=\xi_{c} T_{c}+\left(1-\xi_{c}\right) T_{l}
$$

where the weighting coefficient $\xi_{c}$ is a free parameter whose value is chosen in order to reproduce the thermal properties of the Earth (see Table 1).

To model the formation and evolution of the solid inner core, we need to compare at each time the temperature profile with the iron solidus. We use here the Lindemann law as parameterized by VAL06:

$$
\frac{\partial \log \tau}{\partial \log \rho}=2[\gamma-\delta(\rho)] .
$$

Here, $\delta(\rho) \approx 1 / 3$ and $\gamma$ is an effective Grüneisen parameter that, for simplicity, is assumed to be constant. To integrate this equation, we use the numerical density profile provided by the interior model and the reference values $\rho_{0}=8300 \mathrm{~kg} \mathrm{~m}^{-3}$ (pure iron) and $\tau_{0}=1808 \mathrm{~K}$.

The central temperature $T(r=0, t)$ and the solidus at that point $\tau(r=0)$ are compared at each time step. When $T\left(0, t_{\text {ic }}\right) \approx \tau(0)$ ( $t_{\text {ic }}$ is the time of inner core formation) we turn on the buoyant and latent heat terms in Equations (7) and (8), i.e., set $f_{i}=1$, and continue the integration including these terms. The radius of the inner core at times $t>t_{\text {ic }}$ is obtained by solving the equation proposed by Nimmo (2009) and further developed by Gaidos et al. (2010),

$$
\frac{d R_{\mathrm{ic}}}{d t}=-\frac{D_{c}^{2}}{2 R_{\mathrm{ic}}(\Delta-1)} \frac{1}{T_{c}} \frac{d T_{c}}{d t} .
$$

Here, $\Delta$ is the ratio between the gradient of the solidus (Equation (16)) and the actual temperature gradient $T_{c}(t) f_{c}(r)$ as measured at $R_{\mathrm{ic}}(t)$.

When the core cools down below a given level the outer layers start to stratificate. Here, we model the effect of stratification by correcting the radius and temperature of the core following the prescription by Gaidos et al. (2010). When stratified the effective radius of the core is reduced to $R_{\star}$ (Equation (27) in Gaidos et al. 2010) and the temperature at the core surface is increase to $T_{\star}$ (Equation (28) in Gaidos et al. 2010). The stratification of the core reduces the height of the convective shell which leads to a reduction of the Coriolis force potentially enhancing the intensity of the dynamo-generated magnetic field.

The estimation of the dynamo properties requires the computation of the available convective power $Q_{\text {conv }} . Q_{\text {conv }}$ is calculated here assuming that most of the dissipation occurs at the top of the core. Under this assumption,

$$
Q_{\mathrm{conv}}(t) \approx \Phi(t) T_{c}(t)
$$

where the total entropy $\Phi$ is computed from the entropy balance (Equation (8)) using the solution for the temperature profile $T_{c}(t) f_{c}(r)$.

When $\Phi(t)$ becomes negative, i.e., $E_{l}+E_{s}+E_{g}<E_{k}$ in Equation (8), $Q_{\text {conv }}$ also becomes negative and convection is no longer efficient to transport energy across the outer core. Under this condition, the dynamo is shut down. The integration stops when this condition is fulfilled at a time we label as the dynamo lifetime $t_{\mathrm{dyn}}$.

\subsection{Mantle Thermal Evolution}

One of the novel features of our thermal evolution model is that we treat mantle thermal evolution with a similar formalism as that described before for the metallic core.

The energy balance in the mantle can be written as

$$
Q_{m}=\chi_{r} Q_{r}+Q_{s}+Q_{c}
$$

Here, $Q_{m}$ is the total heat flowing out through the surface boundary (SB), $Q_{r}$ is the heat produced in the decay of radioactive nuclides inside the mantle, $Q_{s}$ is the secular heat, and $Q_{c}$ is the heat coming from the core (Equation (13)).

We use here the standard expressions and parameters for the radioactive energy production as given by Kite et al. (2009). However, in order to correct for the non-homogeneous distribution of radioactive elements in the mantle, we introduce a multiplicative correction factor $\chi_{r}$. Here, we adopt $\chi_{r}=1.253$ that fits the Earth properties well. We have verified that the thermal evolution is not too sensitive to $\chi_{r}$ and have assumed the same value for all planetary masses.

The secular heat in the mantle is computed using an expression analogous to Equation (9). As in the case of the core, we assume that the temperature radial profile does not change during the thermal evolution. Under this assumption, the temperature profile in the mantle can be also written as $T(r, t)=T_{m}(t) f_{m}(r)$. In this case, $T_{m}(t)=T\left(r=R_{p}, t\right)$ is the temperature just below the SB layer (see Figure 1).

Assuming an adiabatical temperature profile in the mantle, we can also write:

$$
f_{m}(r)=\exp \left(\frac{R_{p}^{2}-r^{2}}{D_{m}^{2}}\right)
$$

in analogy to the core temperature profile (Equation (11)). In this expression, $D_{m}$ is the temperature scale height for the mantle which is related to the density scale height $L_{m}$ through $D_{m}^{2}=L_{m}^{2} / \gamma$ (Labrosse 2003). In our simplified model, we take the values of the density at the boundaries of the mantle and analytically obtain an estimate for $L_{m}$ and hence for $D_{m}$.

The energy balance in the mantle is balanced when we independently calculate the heat $Q_{m}$ at the SB as a function of $T_{m}$. In this case, whether or not mobile lids are present plays an important role in determining the efficiency with which the planet gets rid of the heat coming from the mantle. In the mobile lid regime, we assume that the outer layer is fully convective and use the BLT approximation to calculate $Q_{m}$,

$$
Q_{m}^{\mathrm{ML}}=\frac{4 \pi R_{p}^{2} k_{m} \Delta T_{m} \mathrm{Nu}_{m}}{R_{p}-R_{c}}
$$

where $\Delta T_{m}=T_{m}-T_{s}$ is the temperature contrast across the $\mathrm{SB}$ and $T_{s}$ is the surface temperature. Since we are studying the thermal evolution of habitable planets, in all cases we assume $T_{s}=290 \mathrm{~K}$. Planetary interior structures and thermal evolution are not too sensitive to surface temperature. We have verified that the results are nearly the same for the surface temperature in the range of 250-370 K. In the mobile lid regime, $\mathrm{Nu}_{m}$ obeys 


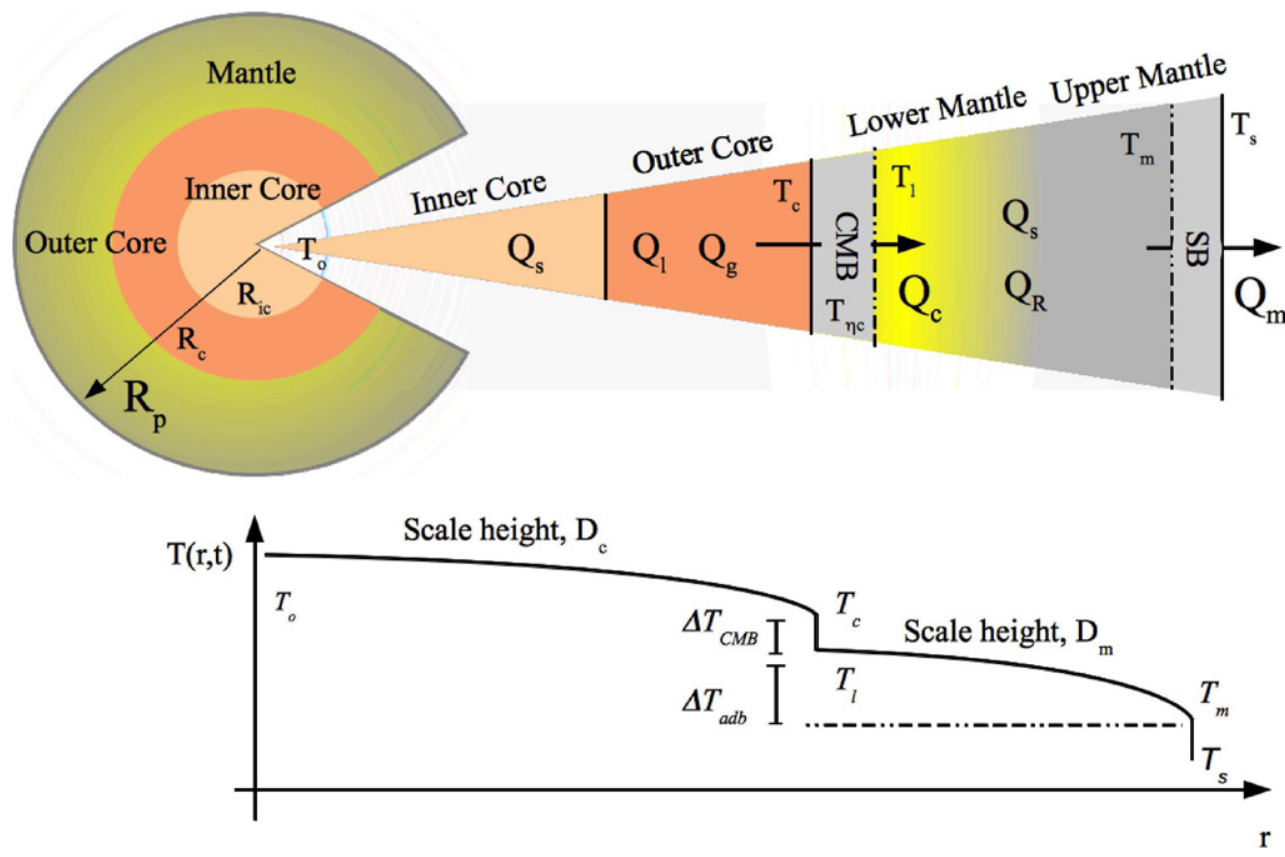

Figure 1. Schematic representation of the planetary interior. In the schematic slice, we depict the main quantities used here to describe the thermal evolution of the planets. The temperature profile depicted below the slice does not use real data. Distances and sizes are not represented with the right scale.

(A color version of this figure is available in the online journal.)

the same relationship with the critical Rayleigh number as in the core. In this case, however, we compute the local Rayleigh number under the assumption of material heated from inside (Gaidos et al. 2010),

$$
\operatorname{Ra}_{m}=\frac{\alpha g \rho^{2} H\left(R_{p}-R_{c}\right)^{5}}{k_{m} \kappa_{m} \eta_{m}},
$$

where $H=\left(Q_{r}+Q_{c}\right) / M_{m}$ is the density of heat inside the mantle, $k_{m}$ and $\kappa_{m}$ are the thermal conductivity and diffusivity, respectively, and $\eta_{m}$ is the upper mantle viscosity.

In the stagnant lid regime, the SB provides a rigid boundary for the heat flux. In this case, we adopt the approximation used by Nimmo \& Stevenson (2000):

$$
Q_{m}^{\mathrm{SL}}=4 \pi R_{p}^{2} \frac{k_{m}}{2}\left(\frac{\rho g \alpha}{\kappa_{m} \eta_{m}}\right)^{1 / 3} \Gamma^{-4 / 3}
$$

Here, $\Gamma \equiv-\partial \ln \eta_{m} / \partial T_{m}$ measures the viscosity dependence on temperature evaluated at the average mantle pressure.

With all these elements at hand the energy balance at Equation (19) is finally transformed into an ordinary differential equation for the upper mantle temperature $T_{m}(t)$,

$$
Q_{m}=\chi_{r} Q_{r}+Q_{c}+C_{m} \frac{d T_{m}}{d t},
$$

where $C_{m}$ is the bulk heat capacity of the upper mantle which is calculated with an expression analogous to Equation (12).

\subsection{Initial Conditions}

In order to solve the coupled differential equations (10), (17), and (23), we need to choose a proper set of initial conditions.

The initial value of the upper mantle temperature is chosen using the prescription by Stamenković et al. (2011). According to this prescription, $T_{m}(t=0)$ is computed by integrating the pressure-dependent adiabatic equation up to the average pressure inside the mantle $\left\langle P_{m}\right\rangle$,

$$
T_{m}(t=0)=\theta \exp \left(\int_{0}^{\left\langle P_{m}\right\rangle} \frac{\gamma_{0}}{K_{s}\left(P^{\prime}\right)} d P^{\prime}\right) .
$$

Here, $\theta=1700 \mathrm{~K}$ is a potential temperature which is assumed to be the same for all planetary masses (Stamenković et al. 2011). Using $T_{m}(t=0)$ and the adiabatic temperature profile, we can obtain the initial lower mantle temperature $T_{l}(t=0)$.

The initial value of the core temperature $T_{c}(t=0)$ is one of the most uncertain parameters in thermal evolution models. Although its actual value or its dependence on the formation history and planetary mass is not known, it is reasonable to start the integration of a simplified thermal evolution model when the core temperature is of the same order as the melting point for $\mathrm{MgSiO}_{3}$ at the lower mantle pressure. A small arbitrary temperature contrast against this reference value (Gaidos et al. 2010; Tachinami et al. 2011) or more complicated massdependent assumptions (Papuc \& Davies 2008) have been used in previous models to set the initial core temperature. We use here a simple prescription that agrees reasonably well with previous attempts and provides a unified expression that could be used consistently for all planetary masses.

According to our prescription, the temperature contrast across the $\mathrm{CMB}$ is assumed to be proportional to the temperature contrast across the whole mantle, i.e., $\Delta T_{\mathrm{CMB}}=\epsilon_{\mathrm{adb}} \Delta T_{\mathrm{adb}}=$ $\epsilon_{\text {adb }}\left(T_{m}-T_{l}\right)$. We have found that the thermal evolution properties of Earth are reproduced when we set $\epsilon_{\mathrm{adb}}=0.7$.

Using this prescription, the initial core temperature is finally calculated using

$$
T_{c}(t=0)=T_{l}+\epsilon_{\mathrm{adb}} \Delta T_{\mathrm{adb}} .
$$

We have observed that the value of the $T_{c}(t=0)$ obtained with this prescription is very close to the perovskite melting temperature at the $\mathrm{CMB}$ for all the planetary masses 
studied here. This result shows that although our criterion is not particularly better physically rooted than those used in previous models, it still relies in just one free parameter, i.e., the ratio of mantle and $\mathrm{CMB}$ contrasts $\epsilon_{\mathrm{adb}}$.

\subsection{Rheological Model}

One of the most controversial aspects and probably the largest source of uncertainties in thermal evolution models is the calculation of the rheological properties of silicates and iron at high pressures and temperatures. A detailed discussion on this important topic is beyond the scope of this paper. An upto-date discussion and analysis of the dependence on pressure and temperature of viscosity in SEs and its influence in thermal evolution can be found in the recent works by Tachinami et al. (2011) and Stamenković et al. (2011).

We use here two different models to calculate viscosity under different ranges of temperatures and pressures. For the high pressures and temperatures of the lower mantle, we use a Nabarro-Herring model (Yamazaki \& Karato 2001),

$$
\eta_{\mathrm{NH}}(P, T)=\frac{R_{g} d^{m}}{D_{0} A m_{\mathrm{mol}}} T \rho(P, T) \exp \left(\frac{b T_{\mathrm{melt}}(P)}{T}\right) .
$$

Here, $R_{g}=8.31 \mathrm{~J} \mathrm{~mol}^{-1} \mathrm{~K}^{-1}$ is the gas constant, $d$ is the grain size, $m$ is the growing exponent, $A$ and $b$ are free parameters, $D_{0}$ is the pre-exponential diffusion coefficient, $m_{\mathrm{mol}}$ is the molar density of perovskite, and $T_{\text {melt }}(P)$ is the melting temperature of perovskite that can be computed with the empirical fit:

$$
T_{\text {melt }}(P)=\sum_{i=0}^{4} a_{i} \cdot P^{i}
$$

All the parameters used in the viscosity model, including the expansion coefficients $a_{i}$ in the melting temperature formula, were taken from the recent work by Stamenković et al. (2011). The Nabarro-Herring formula allows us to compute $\eta_{c}=$ $\eta_{\mathrm{NH}}\left(T_{\eta c}\right)$, where $T_{\eta c}$ is the film temperature computed using the average in Equation (15).

The upper mantle has a completely different mineralogy and it is under the influence of lower pressures and temperatures. Although previous works have used the same model and parameters to calculate viscosity across the whole mantle (Stamenković et al. 2011, for example, use the perovskite viscosity parameters also in the olivine upper mantle), we have found here that using a different rheological model in the upper and lower mantles avoids under- and overestimation, respectively, of the value of viscosity that could have a significant effect on the thermal evolution.

In the upper mantle, we find that using an Arrhenius-type model leads to better estimates of viscosity than that obtained using the Nabarro-Herring model. In the upper mantle, the Nabarro-Herring formula (which is best suited to describe the dependence on viscosity at high pressures and temperatures) leads to huge underestimations of viscosity in that region. In the case of the Earth, this underestimation produces values of the total mantle too high compared to that observed in our planet making impossible to fit the thermal evolution of a simulated Earth.

For the Arrhenius-type formula, we use the same parameterization given by Tachinami et al. (2011):

$$
\eta_{A}(P, T)=\frac{1}{2}\left[\frac{1}{B^{1 / n}} \exp \left(\frac{E^{*}+P V^{*}}{n R_{g} T}\right)\right] \dot{\epsilon}^{(1-n) / n},
$$
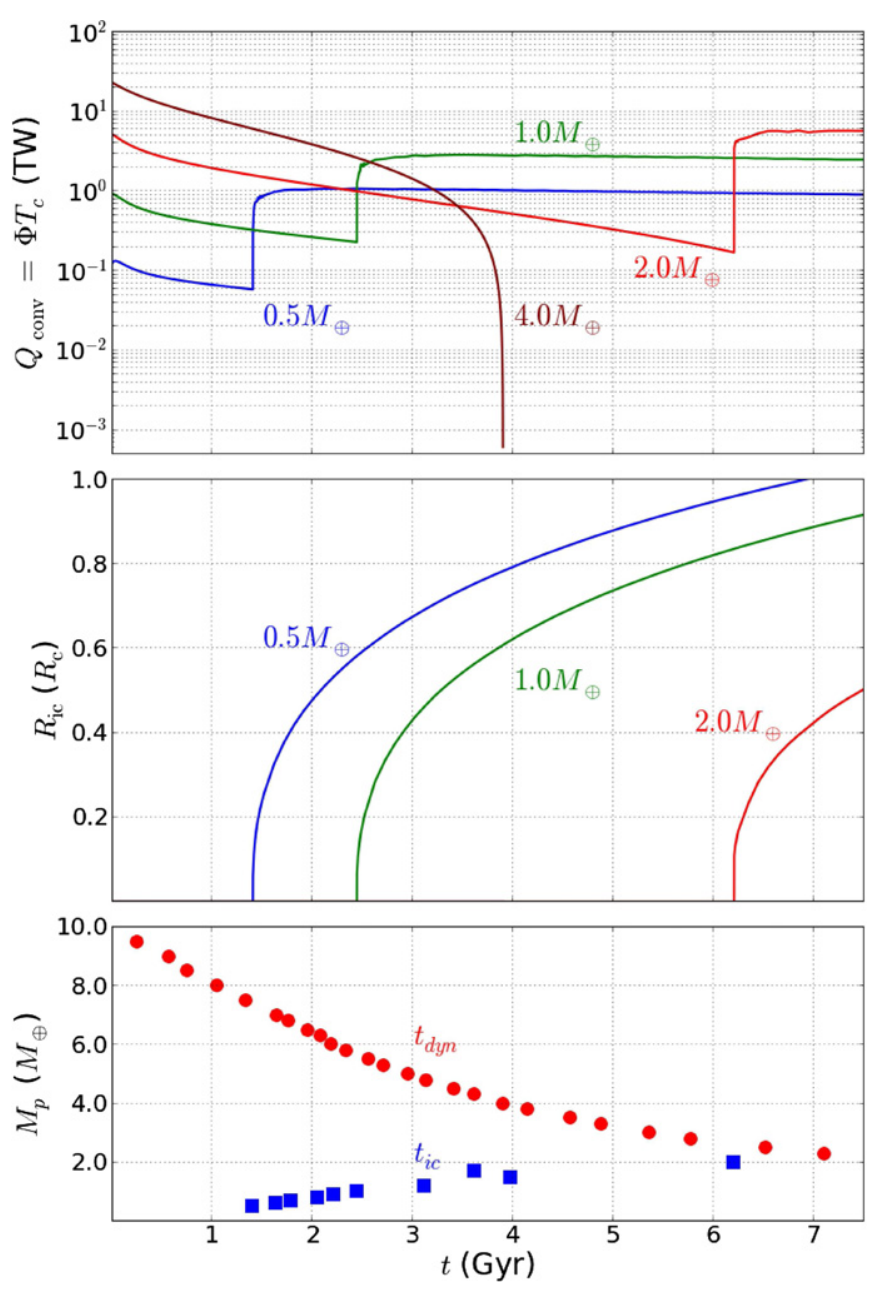

Figure 2. Thermal evolution of TPs with an Earth-like composition (CMF $=$ 0.325 ) using the RTEM (see Table 1). Upper panel: convective power flux $Q_{\text {conv }}$ (see Equation (18)). Middle panel: radius of the inner core $R_{i c}$. Lower panel: time of inner core formation (blue squares) and dynamo lifetime (red circles). In the RTEM, the metallic core is liquid at $t=0$ for all planetary masses. Planets with a mass $M_{p}<M_{\text {crit }}=2.0 M_{\oplus}$ develop a solid inner core before the shutdown of the dynamo while the core of more massive planets remains liquid at least until the dynamo shutdown.

(A color version of this figure is available in the online journal.)

where $\dot{\epsilon}$ is the strain rate, $n$ is the creep index, $B$ is the Barger coefficient, and $E^{*}$ and $V^{*}$ are the activation energy and volume. The values assumed here for these parameters are the same as that given in Table 4 of Tachinami et al. (2011) except for the activation volume whose value we assume here $V^{*}=2.5 \times 10^{-6} \mathrm{~m}^{3} \mathrm{~mol}^{-1}$. Using the formula in Equation (27), the upper mantle viscosity is computed as $\eta_{m}=\eta_{A}\left(\left\langle P_{m}\right\rangle, T_{m}\right)$.

A summary of the parameters used by our interior and thermal evolution models is presented in Table 1 . The values listed in Column 3 define what we will call the reference thermal evolution model (RTEM). These reference values have been mostly obtained by fitting the present interior properties of the Earth and the global features of its thermal, dynamo, and magnetic field evolution (time of inner core formation and present values of $R_{\mathrm{ic}}, Q_{m}$ and surface magnetic field intensity). For the stagnant lid case, we use, as suggested by Gaidos et al. (2010), the values of the parameters that globally reproduce the present thermal and magnetic properties of Venus.

Figure 2 shows the results of applying the RTEM to a set of hypothetical TPs in the mass range $M_{p}=0.5-6 M_{\oplus}$. 


\section{PLANETARY MAGNETIC FIELD}

In recent years, improved numerical experiments have constrained the full set of possible scaling laws used to predict the properties of planetary and stellar convection-driven dynamos (see Christensen 2010 and references therein). It has been found in a wide range of physical conditions that the global properties of a planetary dynamo can be expressed in terms of simple power-law functions of the total convective power $Q_{\text {conv }}$ and the size of the convective region.

One of the most important results of power-based scaling laws is the fact that the volume-averaged magnetic field intensity $B_{\mathrm{rms}}^{2}=(1 / V) \int B^{2} d V$ does not depend on the rotation rate of the planet (Equation (6) in Zuluaga \& Cuartas 2012),

$$
B_{\mathrm{rms}} \approx C_{\mathrm{Brms}} \mu_{0}^{1 / 2} \bar{\rho}_{c}{ }^{1 / 6}(D / V)^{1 / 3} Q_{\mathrm{conv}}^{1 / 3} .
$$

Here, $C_{\mathrm{Brms}}$ is a fitting constant obtained from numerical dynamo experiments and its value is different in the case of dipolar-dominated dynamos, $C_{\mathrm{Brms}}^{\mathrm{dip}}=0.24$, and multipolar dynamos, $C_{\mathrm{Brms}}^{\mathrm{mul}}=0.18 . \bar{\rho}_{c}, D=R_{\star}-R_{\mathrm{ic}}$, and $V=$ $4 \pi\left(R_{\star}^{3}-R_{\text {ic }}^{3}\right) / 3$ are the average density, height, and volume of the convective shell.

The dipolar field intensity at the planetary surface, and hence the dipole moment of the PMF, can be estimated if we have information about the power spectrum of the magnetic field at the core surface. Although we cannot predict the relative contribution of each mode to the total core field strength, numerical dynamos exhibit an interesting property: there is a scalable dimensionless quantity, the local Rossby number $\mathrm{Ro}_{l}^{*}$, that could be used to distinguish dipolar-dominated from multipolar dynamos. The scaling relation for $\mathrm{Ro}_{l}^{*}$ is (Equation (5) in Zuluaga \& Cuartas 2012)

$$
\mathrm{Ro}_{l}^{*}=C_{\mathrm{Ro}_{l}} \bar{\rho}_{c}{ }^{-1 / 6} R_{c}^{-2 / 3} D^{-1 / 3} V^{-1 / 2} Q_{\text {conv }}^{1 / 2} P^{7 / 6} .
$$

Here, $C_{\mathrm{Ro}_{l}}=0.67$ is a fitting constant and $P$ is the period of rotation. It has been found that dipolar-dominated fields arise systematically when dynamos have $\mathrm{Ro}_{l}^{*}<0.1$. Multipolar fields arise in dynamos with values of the local Rossby number close to and larger than this critical value. From Equation (29), we see that in general fast rotating dynamos (low $P$ ) have dipolardominated core fields while slowly rotating ones (large $P$ ) produce multipolar fields and hence fields with a much lower dipole moment.

It is important to stress that the almost independence of $B_{\mathrm{rms}}$ on rotation rate, together with the role that rotation has in the determination of the core field regime, implies that even very slowly rotating planets could have a magnetic energy budget of comparable sized than rapidly rotating planets with similar size and thermal histories. In the former case, the magnetic energy will be redistributed among other multipolar modes rendering the core field more complex in space and probably also in time. Together all these facts introduce a non-trivial dependence of dipole moment on rotation rate very different from that obtained with the traditional scaling laws used in previous works (see, e.g., Grießmeier et al. 2004; Khodachenko et al. 2007). Here, we emphasize a property that was also previously overlooked. Multipolar-dominated dynamos produce magnetic fields that decay more rapidly with distance than dipolar fields and so it is expected that a planet with a multipolar magnetic field will be less protected than those having strongly dipolar fields.

Using the value of $B_{\mathrm{rms}}$ and $\mathrm{Ro}_{l}^{*}$, we can compute the maximum dipolar component of the field at the core surface.
For this purpose, we use an upper bound to the dipolarity fraction $f_{\text {dip }}$ (the ratio of the dipolar component to the total field strength at core surface). Dipolar-dominated dynamos by definition have $f_{\text {dip }} \leqslant f_{\text {dip }}^{\max }=1.0$. The case of reversing dipolar and multipolar dynamos is more complex. Numerical dynamo experiments show that multipolar dynamos have $\mathrm{Ro}_{l}^{*} \gtrsim 0.1$ and $f_{\text {dip }} \lesssim f_{\text {dip }}^{\max }=0.35$. However, to avoid inhomogeneities in the transition region around $\mathrm{Ro}_{l}^{*} \approx 0.1$, we calculate a maximum dipolarity fraction through a "soft step function," $f_{\text {dip }}^{\max }=\alpha+\beta /\left\{\exp \left[\left(\operatorname{Ro}_{l}^{*}-0.1\right) / \delta\right]+1\right\}$ with $\alpha, \beta$, and $\delta$ numerical constants that fit the envelope of the numerical dynamo data (see the upper panel of Figure 1 in Zuluaga \& Cuartas 2012).

To connect this ratio to the volumetric-averaged magnetic field $B_{\text {rms }}$, we use the volumetric dipolarity fraction $b_{\text {dip }}$ that is found, as shown by numerical experiments, conveniently related to the maximum value of $f_{\text {dip }}$ through Equation (12) in Zuluaga \& Cuartas (2012),

$$
b_{\mathrm{dip}}^{\min }=c_{\mathrm{bdip}} f_{\mathrm{dip}}^{\max -11 / 10},
$$

where $c_{\text {bdip }} \approx 2.5$ is again a fitting constant. It is important to notice here that the exponent $11 / 10$ is the ratio of the smallest integers close to the numerical value of the fitting exponent (see Figure 1 in Zuluaga \& Cuartas 2012). We use this convention following Olson \& Christensen (2006).

Finally by combining Equations (28)-(30), we can compute an upper bound to the dipolar component of the field at the CMB:

$$
B_{c}^{\text {dip }} \lesssim \frac{1}{b_{\text {dip }}^{\min }} B_{\mathrm{rms}}=\frac{f_{\text {dip }}^{\max 11 / 10}}{c_{\text {bdip }}} B_{\mathrm{rms}}
$$

The surface dipolar field strength is estimated using

$$
B_{p}^{\operatorname{dip}}\left(R_{p}\right)=B_{c}^{\operatorname{dip}}\left(\frac{R_{p}}{R_{c}}\right)^{3}
$$

and finally the total dipole moment is calculated using Equation (1) for $r=R_{p}$.

It should be emphasized that the surface magnetic field intensity determined using Equation (32) overestimates the PMF dipolar component. The actual field could be much more complex spatially and the dipolar component could be lower. As a consequence, our model can only predict the maximum level of protection that a given planet could have from a dynamogenerated intrinsic PMF.

The results of applying the RTEM to calculate the properties of the magnetic field of TPs in the mass range $0.5-4.0 M_{\oplus}$ using the scaling laws in Equations (28), (29), and (31) are summarized in Figures 3 and 4. In Figure 3, we show the local Rossby number, the maximum dipolar field intensity, and the dipole moment as a function of time computed for planets with different masses and two different periods of rotation ( $P=1$ day and $P=2$ days). This figure shows the effect that rotation has on the evolution of dynamo geometry and hence in the maximum attainable dipolar field intensity at the planetary surface. In Figure 4, we have summarized in mass-period $(M-P)$ diagrams (Zuluaga \& Cuartas 2012) the evolution of the dipole moment for planets with long-lived dynamos. We see that for periods lower than one day and larger than five to seven days the dipole moment is nearly independent of rotation. Slowly rotating planets have a non-negligible dipole moment that is systematically larger for more massive planets. 

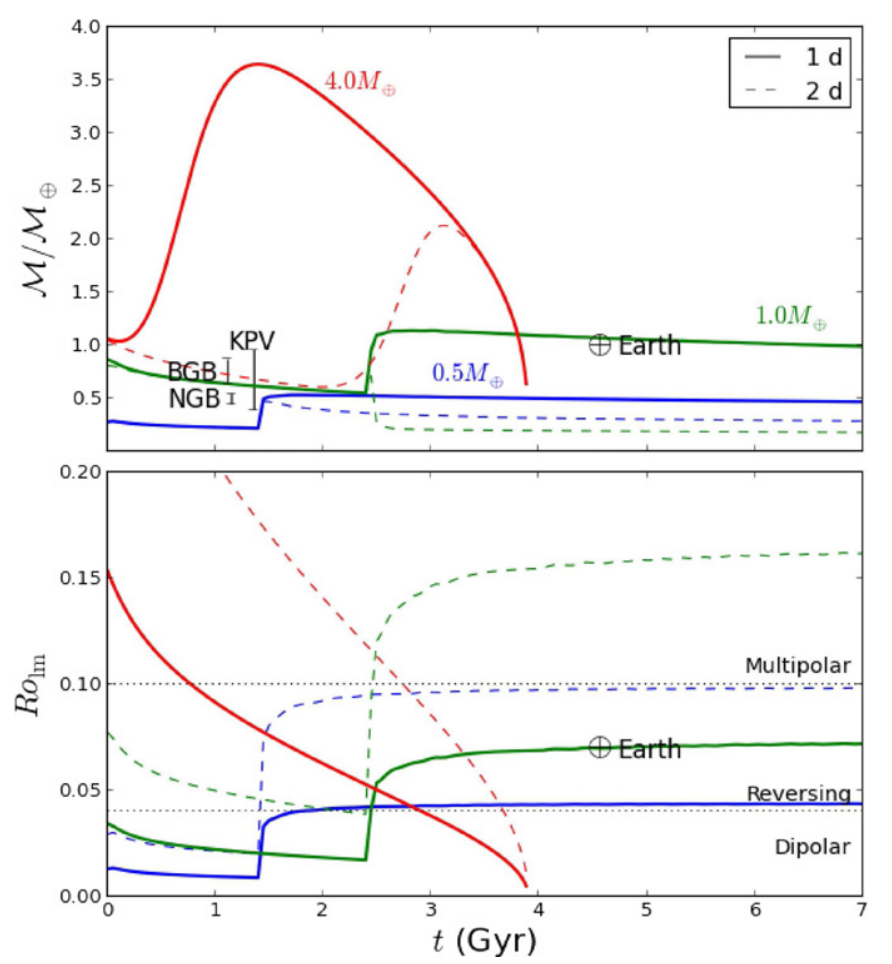

Figure 3. PMF properties predicted using the RTEM and Equations (28), (29), and (31) for TPs $0.5-4.0 M_{\oplus}$. We plot the local Rossby number (lower panel), the maximum surface dipole field (middle panel), and the maximum dipole moment (upper panel). We included the present values of the geodynamo (" $\oplus$ " symbol) and three measurements of paleomagnetic intensities (error bars) at 3.2 and 3.4 Gyr ago (Tarduno et al. 2010). We compare the magnetic properties for two periods of rotation, one day (solid curves), and two days (dashed curves). The effect of a larger period of rotation is more significant at early times in the case of massive planets $\left(M_{p} \gtrsim 2 M_{\oplus}\right)$ and at late times for lower mass planets. (A color version of this figure is available in the online journal.)

\section{PLANET-STAR INTERACTION}

The PMF properties constrained using the thermal evolution model and the dynamo-scaling laws are not enough to evaluate the level of magnetic protection of a potentially habitable TP. We also need to estimate the magnetosphere and stellar properties (stellar wind and luminosity) as a function of time in order to properly assess the level of star-planet interaction.

Since the model developed in previous sections provides only the maximum intensity of the PMF, we will be interested here in constraining the magnetosphere and stellar properties from below, i.e., to find the lower level of "stellar aggression" for a given star-planet configuration. Combining the upper bounds of PMF properties and the lower bounds for the star-planet interaction will produce an overestimation of the overall magnetic protection of a planet. If, using this model, a given star-planet configuration is not suitable to provide enough magnetic protection to the planet, the actual case should be much worse. If, on the other hand, our upper limit approach predicts a high level of magnetic protection, the actual case could still be that of an unprotected planet. Therefore, our model is capable of predicting which planets will be unprotected but less able to predict which ones will actually be protected.

\subsection{The Habitable Zone (HZ) and Tidally Locking Limits}

The surface temperature and hence "first-order" habitability of a planet depends on three basic factors: (1) the fundamental
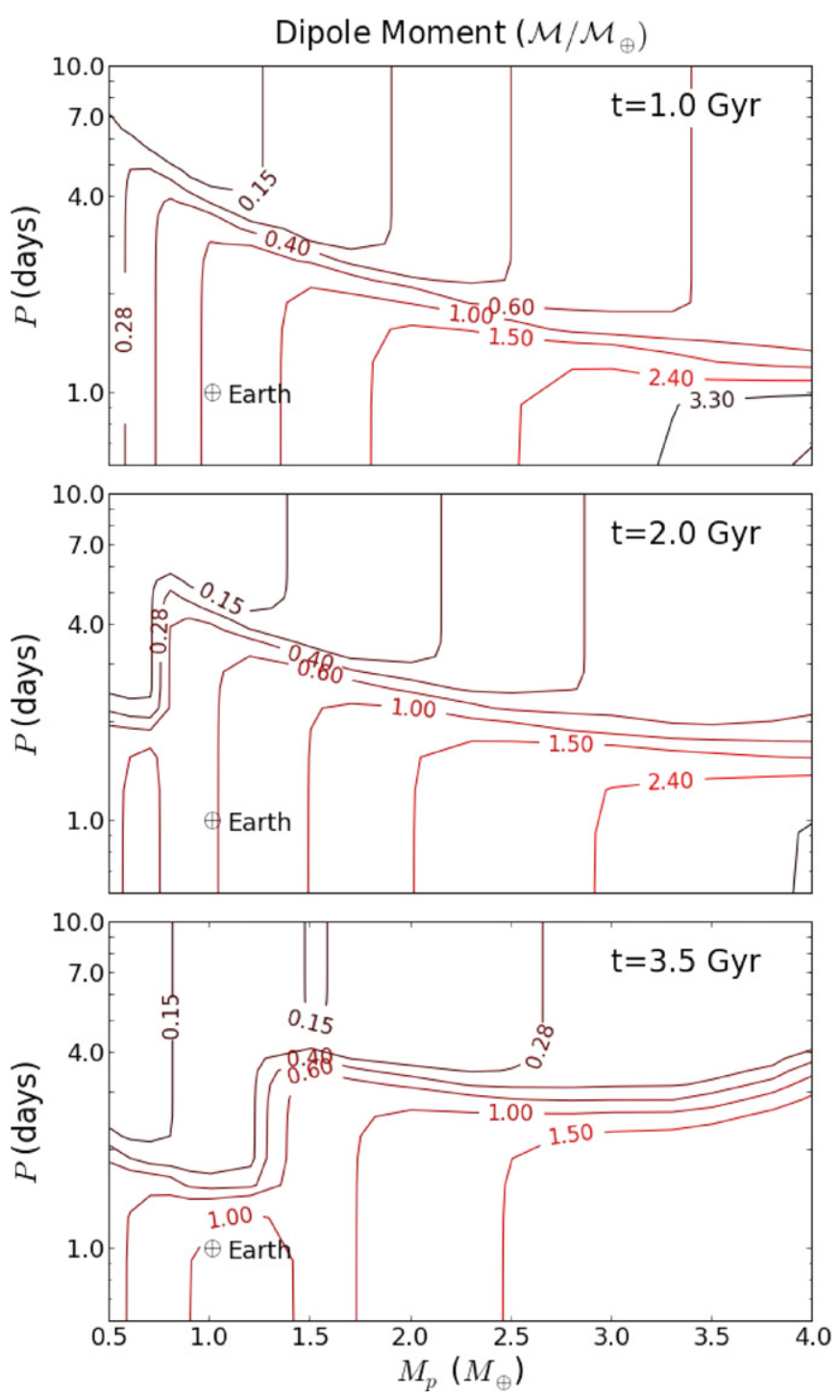

Figure 4. Mass-period $(M-P)$ diagrams of the dipole moment for long-lived planetary dynamos using the RTEM. Three regimes are identified (Zuluaga \& Cuartas 2012): rapid rotating planets $(P \lesssim 1$ day), dipole moments are large and almost independent of rotation rate; slowly rotating planets ( 1 day $\lesssim$ $P \lesssim 5$ day), dipole moments are intermediate in value and highly dependent on rotation rate; and very slowly rotating planets ( $P \gtrsim 5-10$ days), small but non-negligible rotation-independent dipole moments. For $\left(M_{p}<2 M_{\oplus}\right)$, the shape of the dipole-moment contours is determined by $t_{\mathrm{ic}}$.

(A color version of this figure is available in the online journal.)

properties of the star (luminosity $L_{\star}$, effective temperature $T_{\star}$, and radius $R_{\star}$ ), (2) the average star-planet distance (distance to the HZ), and (3) the commensurability of planetary rotation and orbital period (tidal locking). These properties should be properly modeled in order to assess the degree of star-planet interaction which is critical for determining the magnetic protection.

The basic properties of main-sequence stars of different masses and metallicities have been studied for decades and are becoming critical for assessing the actual properties of newly discovered exoplanets. The case of low-mass mainsequence stars (GKM) are particularly important for providing the properties of the stars with the highest potential to harbor habitable planets with evolved and diverse biospheres.

In this work, we will use the theoretical results of Baraffe et al. (1998, hereafter BAR98) that predict the evolution of different 


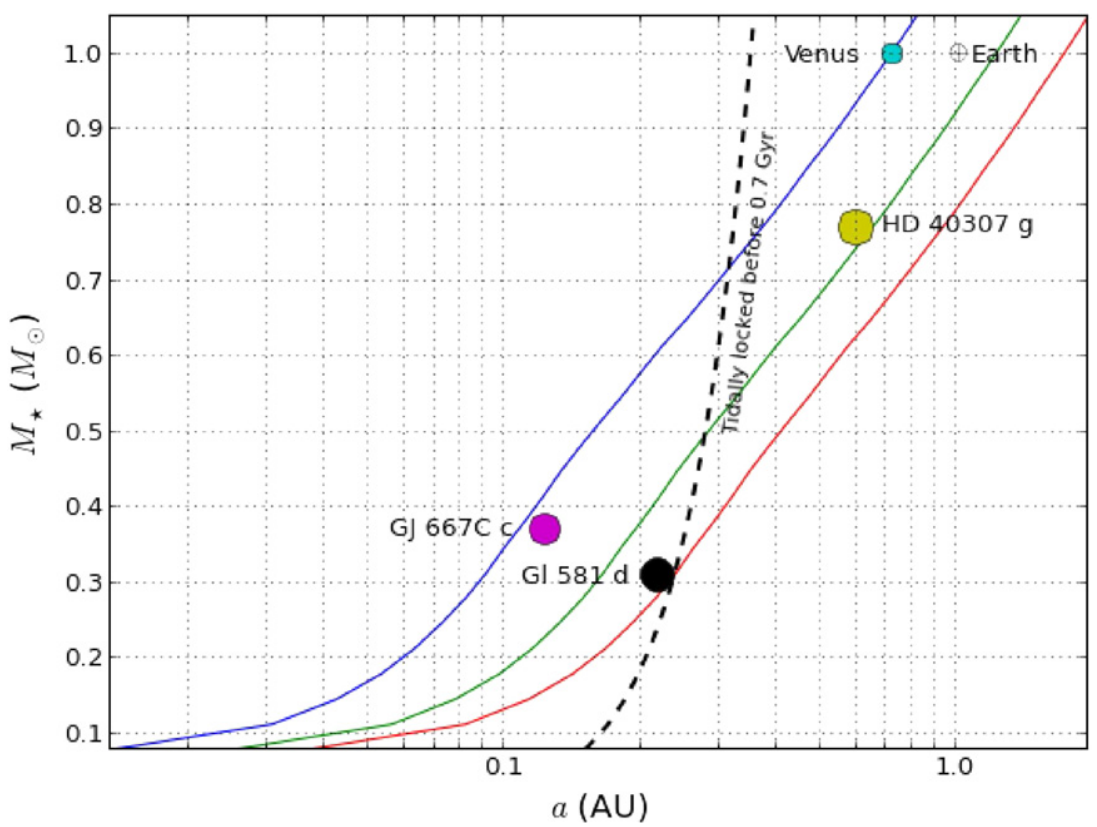

Figure 5. HZ limits corresponding to the conservative criteria of recent Venus and early Mars according to the updated limits estimated by Kopparapu et al. (2013). Stellar properties are computed at $\tau=3 \mathrm{Gyr}$ using the models by Baraffe et al. (1998). Planets at distances below the dashed line would be tidally locked before 0.7 Gyr (Peale 1977). The location of Earth, Venus, and the potentially habitable extrasolar system planets GL 581d, GJ 667Cc, and HD 40307g are also included. (A color version of this figure is available in the online journal.)

Table 2

Properties of the SEs Already Discovered Inside the HZ of Their Host Stars

\begin{tabular}{|c|c|c|c|c|c|c|c|c|c|c|}
\hline Planet & $\begin{array}{c}M_{p} \\
\left(M_{\oplus}\right)\end{array}$ & $\begin{array}{c}R_{p} \\
\left(R_{\oplus}\right)\end{array}$ & $\begin{array}{c}\mathrm{a} \\
(\mathrm{AU})\end{array}$ & $\begin{array}{c}P_{o} \\
\text { (days) }\end{array}$ & $e$ & S-type & $\begin{array}{c}M_{\star} \\
\left(M_{\odot}\right)\end{array}$ & $\begin{array}{l}\text { Age } \\
\text { (Gyr) }\end{array}$ & Tid. Locked & Refs. \\
\hline Earth & 1.0 & 1.0 & 1.0 & 365.25 & 0.016 & $\mathrm{G} 2 \mathrm{~V}$ & 1.0 & 4.56 & No & $\cdots$ \\
\hline Venus & 0.814 & 0.949 & 0.723 & 224.7 & 0.007 & $\mathrm{G} 2 \mathrm{~V}$ & 1.0 & 4.56 & Probably & $\ldots$ \\
\hline GJ $667 \mathrm{Cc}$ & 4.545 & $1.5^{*}$ & 0.123 & 28.155 & $<0.27$ & $\mathrm{M} 1.25 \mathrm{~V}$ & 0.37 & $>2.0$ & Yes & (1) \\
\hline GL 581d & 6.038 & $1.6^{*}$ & 0.22 & 66.64 & 0.25 & M3V & 0.31 & $4.3-8.0$ & Yes & (2), (3) \\
\hline $\mathrm{HD} 40307 \mathrm{~g}$ & 7.1 & $1.7^{*}$ & 0.6 & 197.8 & 0.29 & $\mathrm{~K} 2.5 \mathrm{~V}$ & 0.77 & 4.5 & No & (4) \\
\hline
\end{tabular}

Notes. For reference purposes, the properties of Venus and the Earth are also included. Values of radii marked with an “*” are unknown and were estimated using the mass-radius relation for planets with the same composition as the Earth, i.e., $R_{p}=R_{\oplus}\left(M_{p} / M_{\oplus}\right)^{0.27}$ (VAL06).

References. (1) Bonfils et al. 2011; (2) Udry et al. 2007; (3) Mayor et al. 2009; (4) Tuomi et al. 2012.

metallicities main-sequence GKM stars. We have chosen from that model those results corresponding to the case of solar metallicity stars. We have disregarded the fact that the basic stellar properties actually evolve during the critical period where magnetic protection will be evaluated, i.e., $t=0.5-3$ Gyr. To be consistent with the purpose of estimating upper limits of magnetic protection, we took the stellar properties as provided by the model at the highest end of the time interval, i.e., $t=3$ Gyr. Since luminosity increases with time in GKM stars, this assumption guarantees the largest distance of the $\mathrm{HZ}$ and hence the lowest effects of the stellar insolation and the stellar wind.

In order to estimate the HZ limits, we use the recently updated values calculated by Kopparapu et al. (2013). In particular, we use the interpolation formula in Equation (2) and coefficients in Table (2) to compute the most conservative limits of recent Venus and early Mars. The limits calculated for the stellar properties assumed here are depicted in Figure 5.

The orbital and rotational properties of planets at close-in orbits are strongly affected by gravitational and tidal interactions with the host star. Tidal torque dampens the primordial rotation and axis tilt leaving the planet in a final resonant equilibrium where the period of rotation $P$ becomes commensurable with the orbital period $P_{o}$,

$$
P: P_{o}=n: 2 \text {. }
$$

Here, $n$ is an integer larger than or equal to 2. The value of $n$ is determined by multiple dynamic factors, the most important being the orbital planetary eccentricity (Leconte et al. 2010; Ferraz-Mello et al. 2008; Heller et al. 2011). In the solar system, the tidal interaction between the Sun and Mercury has trapped the planet in a 3:2 resonance. In the case of GL 581d, detailed dynamic models predict a resonant 2:1 equilibrium state (Heller et al. 2011), i.e., the rotation period of the planet is a half of its orbital period.

Although in general estimating the time required for the "tidal erosion" is very hard given the large uncertainties in the key physical parameters involved (see Heller et al. 2011 for a detailed discussion), the maximum distance $a_{\text {tid }}$ at which a solid planet in a circular orbit becomes tidally locked before a given time $t$ can be roughly estimated by (Peale 1977)

$$
a_{\text {tid }}(t)=0.5 \mathrm{AU}\left[\frac{\left(M_{\star} / M_{\odot}\right)^{2} P_{\text {prim }}}{Q}\right]^{1 / 6} t^{1 / 6} .
$$


Here, the primordial period of rotation $P_{\text {prim }}$ should be expressed in hours, $t$ in Gyr, and $Q$ is the dimensionless dissipation function. For the purposes of this work, we assume a primordial period of rotation $P_{\text {prim }}=17 \mathrm{hr}$ (Varga et al. 1998; Denis et al. 2011) and a dissipation function $Q \approx 100$ (Henning et al. 2009; Heller et al. 2011).

In Figure 5, we summarize the properties of solar metallicity GKM main-sequence stars provided by the BAR98 model and the corresponding limits of the $\mathrm{HZ}$ and tidal locking maximum distance. The properties of the host stars of the potentially habitable SEs already discovered, GL 581d, GJ 667Cc, and HD $40307 \mathrm{~g}$, are also highlighted in this figure.

\subsection{Stellar Wind}

The stellar wind and CRs pose the highest risks for a magnetically unprotected potentially habitable TP. The dynamic pressure of the wind is able to obliterate an exposed atmosphere, especially during the early phase of stellar evolution (Lammer et al. 2003), and energetic stellar CRs could pose a serious risk to any form of surface life directly exposed to them (Grießmeier et al. 2005).

The last step in order to estimate the magnetospheric properties and hence the level of magnetic protection is predicting the stellar wind properties for different stellar masses and as a function of planetary distance and time.

There are two simple models used to describe the spatial structure and dynamics of the stellar wind: the pure hydrodynamical model originally developed by Parker (1958) that describes the wind as a non-magnetized, isothermal, and axially symmetric flux of particles (hereafter Parker's model), and the more detailed albeit simpler magneto-hydrodynamic model originally developed by Weber \& Davis (1967) that takes into account the effects of stellar rotation and treats the wind as a magnetized plasma.

It has been shown that Parker's model reliably describes the properties of the stellar winds in the case of stars with periods of rotation of the same order as the present solar value, i.e., $P \sim 30$ days (Preusse et al. 2005). However, for rapidly rotating stars, i.e., young stars and/or active dM stars, the isothermal model underestimates the stellar wind properties by almost a factor of two (Preusse et al. 2005). For the purposes of scaling the properties of the planetary magnetospheres (Equations (3)-(6)), an underestimation of the stellar wind dynamic pressure of that size will give us values of the key magnetospheric properties that will be off by $10 \%-40 \%$ of the values given by more detailed models. Magnetopause fields that have the largest uncertainties will be underestimated by $\sim 40 \%$, while standoff distances and polar cap areas will be, respectively, under- and overestimated by just $\sim 10 \%$.

According to Parker's model, the stellar wind average particle velocity $v$ at distance $d$ from the host star is obtained by solving Parker's wind equation (Parker 1958):

$$
u^{2}-\log u=4 \log \rho+\frac{4}{\rho}-3,
$$

where $u=v / v_{c}$ and $\rho=d / d_{c}$ are the velocity and distance normalized with respect to $v_{c}=\sqrt{k_{B} T / m}$ and $d_{c}=$ $G M_{\star} m /\left(4 k_{B} T\right)$ which are, respectively, the local sound velocity and the critical distance where the stellar wind becomes subsonic. $T$ is the temperature of the plasma which, in the isothermal case, is assumed to be constant at all distances and equal to the temperature of the stellar corona. $T$ is the only free parameter controlling the velocity profile of the stellar wind.
The number density $n(d)$ is calculated from the velocity using the continuity equation:

$$
n(d)=\frac{\dot{M}_{\star}}{4 \pi d^{2} v(d) m} .
$$

Here, $\dot{M}_{\star}$ is the stellar mass-loss rate, which is a free parameter in the model.

To calculate the evolution of the stellar wind, we need a way to estimate the evolution of the coronal temperature $T$ and the mass-loss rate $\dot{M}_{\star}$.

Using observational estimates of the stellar mass-loss rate (Wood et al. 2002) and theoretical models for the evolution of the stellar wind velocity (Newkirk 1980), Grießmeier et al. (2004) and Lammer et al. (2004) developed semiempirical formulae to calculate the evolution of the long-term-averaged number density and velocity of the stellar wind for main-sequence stars at a given reference distance (1 AU):

$$
\begin{aligned}
& v_{1 \mathrm{AU}}(t)=v_{0}\left(1+\frac{t}{\tau}\right)^{\alpha_{v}} \\
& n_{1 \mathrm{AU}}(t)=n_{0}\left(1+\frac{t}{\tau}\right)^{\alpha_{n}} .
\end{aligned}
$$

Here, $\alpha_{v}=-0.43, \alpha_{n}=-1.86 \pm 0.6$, and $\tau=25.6 \mathrm{Myr}$ (Grießmeier et al. 2009). The parameters $v_{0}=3971 \mathrm{~km} \mathrm{~s}^{-1}$ and $n_{0}=1.04 \times 10^{11} \mathrm{~m}^{-3}$ are estimated from the present longterm averages of the solar wind as measured at the distance of the Earth $n\left(4.6 \mathrm{Gyr}, 1 \mathrm{AU}, 1 M_{\odot}\right)=6.59 \times 10^{6} \mathrm{~m}^{-3}$ and $v\left(4.6 \mathrm{Gyr}, 1 \mathrm{AU}, 1 M_{\odot}\right)=425 \mathrm{~km} \mathrm{~s}^{-1}$ (Schwenn 1990).

Using these formulae, Grießmeier et al. (2007a) devised a clever way to estimate consistently $T(t)$ and $\dot{M}_{\star}(t)$ in Parker's model and hence we are able predict the stellar wind properties as a function of $d$ and $t$. For the sake of completeness, we summarize this procedure here. For further details, see Section 2.4 in Grießmeier et al. (2007a).

For a stellar mass $M_{\star}$ and time $t$, the velocity of the stellar wind at $d=1 \mathrm{AU}, v_{1 \mathrm{AU}}$, is calculated using Equation (37). Replacing this velocity in Parker's wind equation for $d=1 \mathrm{AU}$, we numerically find the temperature of the corona $T(t)$. This parameter is enough to provide the whole velocity profile $v\left(t, d, M_{\star}\right)$ at time $t$. To compute the number density, we need the mass-loss rate for this particular star and at this time. Using the velocity and number density calculated from Equations (37) and (38), the mass-loss rate for the Sun $\dot{M}_{\odot}$ at time $\mathrm{t}$ and $d=1$ AU can be obtained:

$$
\dot{M}_{\odot}(t)=4 \pi(1 \mathrm{AU})^{2} m n_{1 \mathrm{AU}}(t) v_{1 \mathrm{AU}}(t) .
$$

Assuming that the mass-loss rate scales simply with the stellar surface area, i.e., $\dot{M}_{\star}(t)=\dot{M}_{\odot}(t)\left(R_{\star} / R_{\odot}\right)^{2}$, the value of $\dot{M}_{\star}$ can finally be estimated. Using $v\left(t, d, M_{\star}\right)$ and $\dot{M}_{\star}$ in the continuity equation (36), the number density of the stellar wind $n\left(t, d, M_{\star}\right)$ is finally obtained.

The value of the stellar wind dynamic pressure $P_{\text {dyn }}\left(t, d, M_{\star}\right)=m n\left(t, d, M_{\star}\right) v\left(t, d, M_{\star}\right)^{2}$ inside the HZ of four different stars as computed using the procedure described previously is plotted in Figure 6.

It is important to stress here that for stellar ages $t \lesssim 0.7 \mathrm{Gyr}$ the semiempirical formulae in Equations (37) and (38) are not longer reliable (Grießmeier et al. 2007a). These equations are based on the empirical relationship observed between the X-ray surface flux and the mass-loss rate $\dot{M}_{\star}$ (Wood et al. 


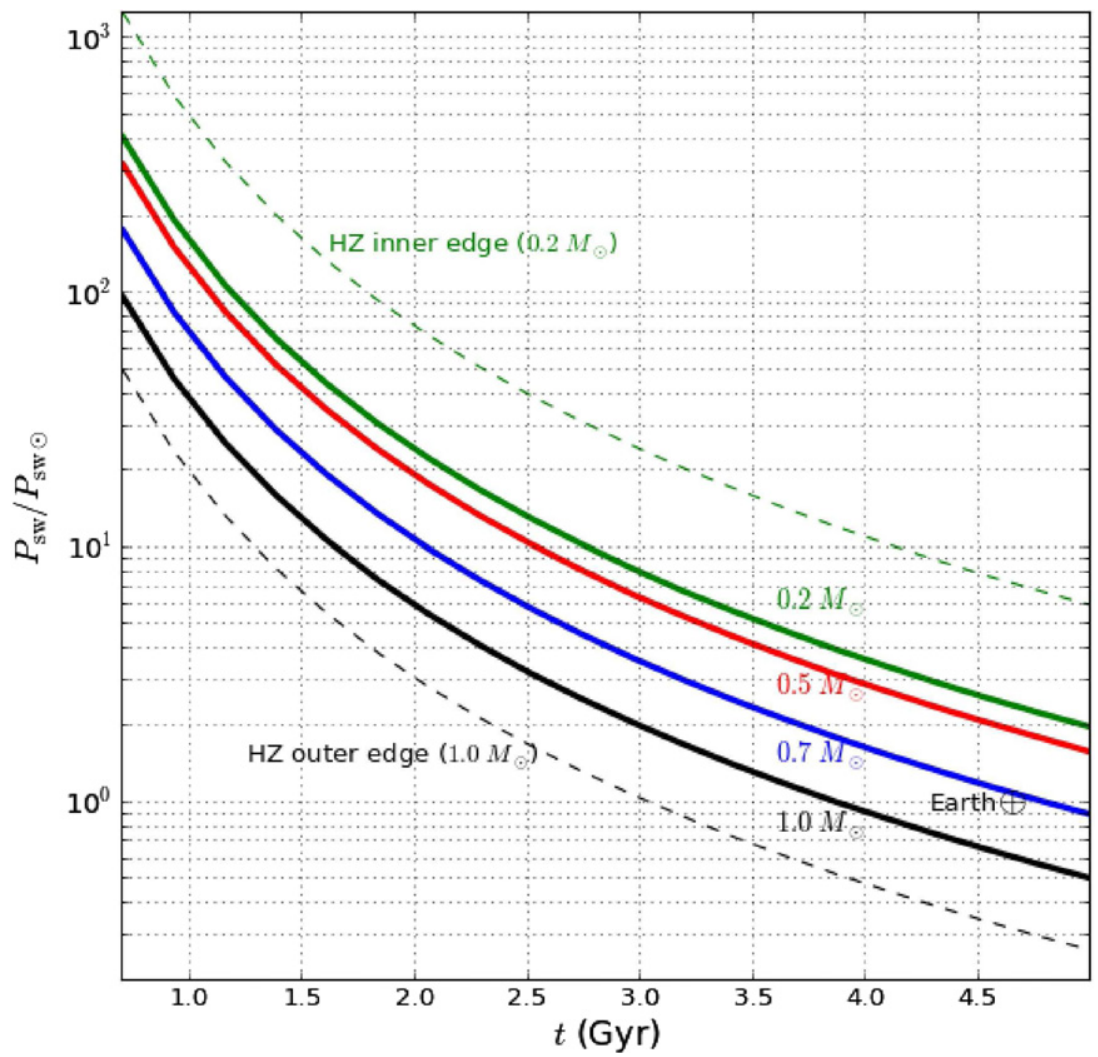

Figure 6. Evolution of the stellar wind dynamic pressure at the center of the $\mathrm{HZ}$ for a selected set of stellar masses. The reference average solar wind pressure is $P_{\mathrm{SW} \odot}=1.86 \mathrm{nPa}$. The dashed curves indicate the value of the stellar wind pressure at the inner and outer edges of the $\mathrm{HZ}$ around stars with $0.2 M_{\odot}$ and $1.0 M_{\odot}$, respectively. The $\mathrm{HZ}$ limits where the pressure was calculated are assumed to be static and equal to those at $\tau=3 \mathrm{Gyr}$. Stellar wind pressure at $t<0.7 \mathrm{Gyr}$ computed with the semiempirical model used in this work is too uncertain and was not plotted.

(A color version of this figure is available in the online journal.)

2002, 2005) which has been reliably obtained only for ages $t \gtrsim 0.7$ Gyr. However, Wood et al. (2005) have shown that an extrapolation of the empirical relationship to earlier times overestimates the mass-loss rate by a factor of 10-100. At times $t \lesssim 0.7 \mathrm{Gyr}$ and over a given magnetic activity threshold, the stellar wind of main-sequence stars seems to be inhibited (Wood et al. 2005). Therefore, the limit placed by observations at $t \approx 0.7 \mathrm{Gyr}$ is not simply an observational constraint but could mark the time where the early stellar wind also reaches a maximum (J. L. Linsky 2012, private communication). This fact suggests that at early times the effect of the stellar wind on the planetary magnetosphere is much lower than normally assumed. Hereafter, we will assume that intrinsic PMF is strong enough to protect the planet, at least until the maximum of the stellar wind is reached at $t \approx 0.7 \mathrm{Gyr}$, and focus on the stellar wind and magnetosphere properties for times larger than this.

\section{RESULTS}

Using the results of our RTEM, the power-based scaling laws for dynamo properties, and the properties of the stellar insolation and stellar wind pressure, we have calculated the magnetosphere properties of EPs and SEs in the HZ of different main-sequence stars. We have performed these calculations for hypothetical TPs in the mass range $0.5-6 M_{\oplus}$ and for the potentially habitable planets already discovered GL 581d, GJ 667Cc, and HD 40307g (see Table 2). The case of the Earth and a habitable Venus have also been studied for references purposes.

To include the effect of rotation in the properties of the PMF, we have assumed that planets in the $\mathrm{HZ}$ of late $\mathrm{K}$ and $\mathrm{dM}$ stars
$\left(M<0.7 M_{\odot}\right)$ are tidally locked at times $t<0.7 \mathrm{Gyr}(n=$ 2 in Equation (33), see Figure 5). Planets around $\mathrm{G}$ and early $\mathrm{K}$ stars $\left(M \gtrsim 0.7 M_{\odot}\right)$ will be assumed to have primordial periods of rotation that we chose in the range 1-100 days as predicted by models of planetary formation (Miguel \& Brunini 2010).

Figures 7 and 8 show the evolution of magnetosphere properties for tidally locked and unlocked potentially habitable planets, respectively. In all cases, we have assumed that the planets are in the middle of the $\mathrm{HZ}$ of their host stars.

Even at early times, tidally locked planets of arbitrary masses have non-negligible magnetosphere radii $R_{S}>1.5 R_{p}$. Previous estimates of the standoff distances for tidally locked planets are much lower than the values reported here. As an example, Khodachenko et al. (2007) place the standoff distances well below $2 R_{p}$, even under mild stellar wind conditions (see Figure 4 in their work) and independent of planetary mass and age. In contrast, our model predicts standoff distances for tidally locked planets in the range of $2-6 R_{p}$ depending on planetary mass and stellar age. The differences between both predictions arise mainly from the underestimation of the dipole moment for slowly rotating planets found in these works. Thermal evolution and the dependency on planetary mass of the PMF properties are responsible for the rest of the discrepancies in previous estimates of the magnetosphere properties.

Though tidally locked planets seem to have larger magnetospheres than previously expected, they still have large polar caps, a feature that was previously overlooked. As a consequence of this fact, well protected atmospheres, i.e., atmospheres that lie well inside of the magnetosphere cavity (hereafter magnetized 


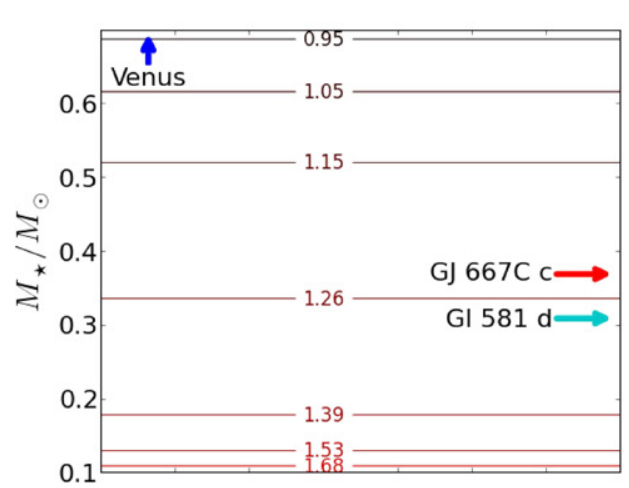

Magnetopause Field $B_{m p}(\mu \mathrm{T})$
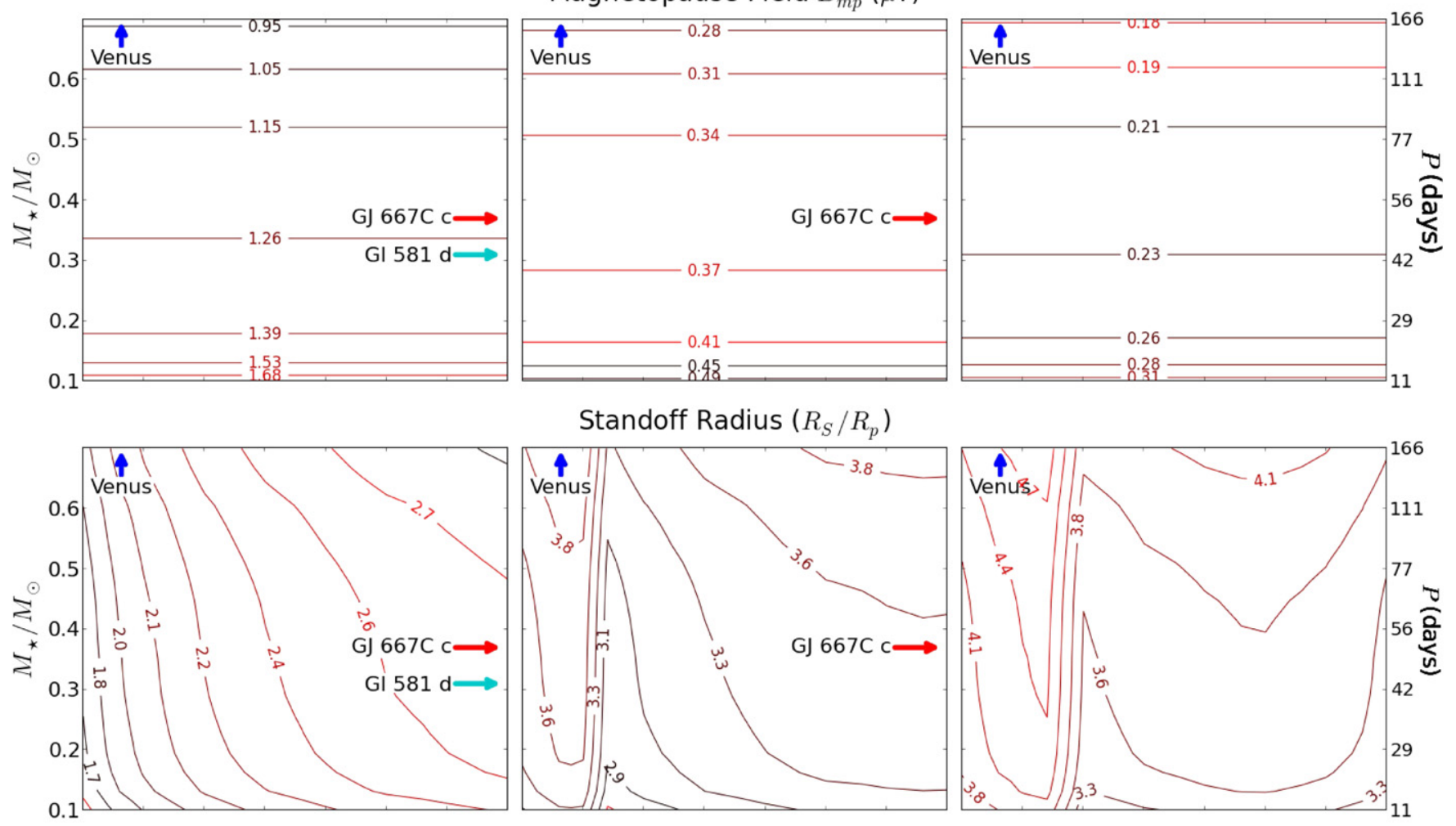

Standoff Radius $\left(R_{S} / R_{p}\right)$
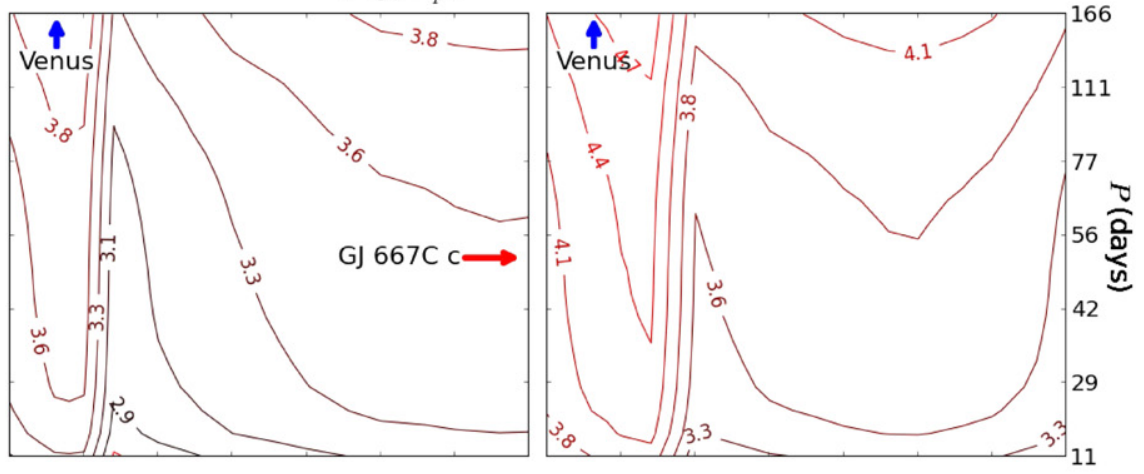

Polar Cap Area, $A_{p c} / A_{p}(\%)$
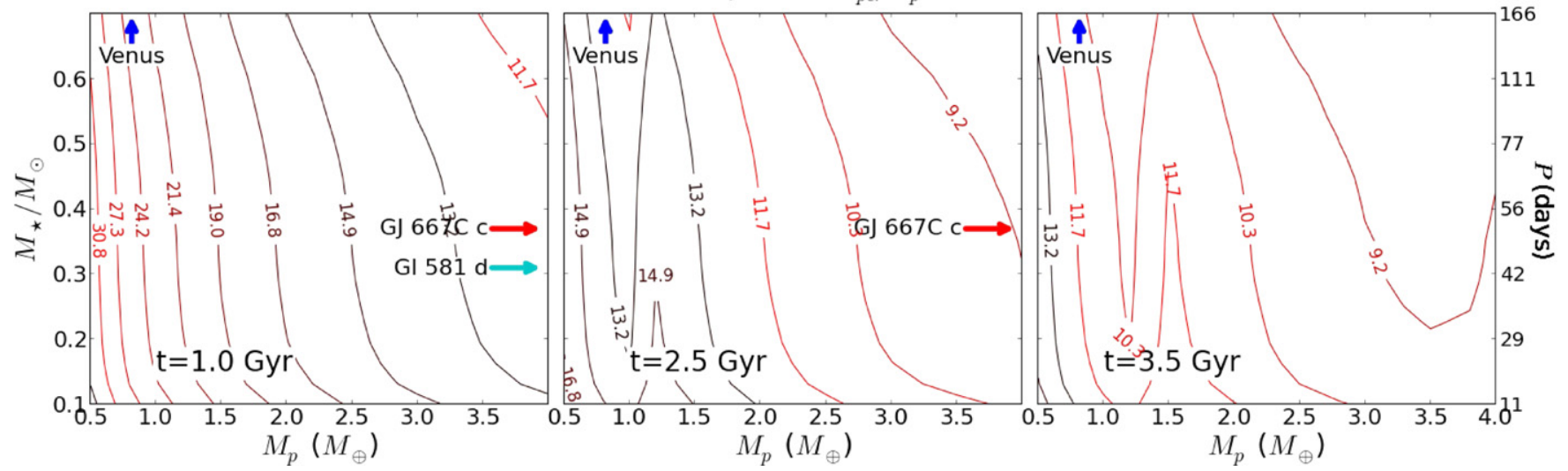

Figure 7. Evolution of the magnetopause field (upper row), standoff distance (middle row), and polar cap area (lower row) of tidally locked (slow rotating) planets around late $\mathrm{dK}$ and $\mathrm{dM}$ stars. The rotation of each planet is assumed to be equal to the orbital period at the middle of the HZ (see the values in the rightmost vertical axes). The value of the magnetosphere properties returned by the contour lines in these plots could be an under- or an overestimation of these properties according to the position of a planet inside the HZ. In the case of GL 581d (GJ 667Cc), which is located in the outer (inner) edge of the HZ, the magnetopause field and polar cap area are overestimated (underestimated) while the standoff distance is underestimated (overestimated).

(A color version of this figure is available in the online journal.)

planets), could have more than $15 \%$ of their surface area exposed to open field lines where thermal and non-thermal processes could efficiently remove atmospheric gases. Moreover, our model predicts that these planets would have multipolar PMF which contributes to an increase of the atmospheric area open to the interplanetary and magnetotail regions (Siscoe \& Crooker 1976). Then the exposition of magnetized planets to harmful external effects would be a complex function of the standoff distance and the polar cap area.

Overall, magnetic protection improves with time. As the star evolves, the dynamic pressure of the stellar wind decreases more rapidly than the dipole moment (see Figures 4 and 6). As a consequence, the standoff distance grows in time and the polar cap shrinks. However, with the reduction in time of the stellar wind pressure, the magnetopause field is also reduced, which can affect the incoming flux of $\mathrm{CR}$ at late times.

The sinuous shape of the contour lines in the middle and lower rows is a byproduct of the inner core solidification in planets with $M_{p}<2 M_{\oplus}$. Critical boundaries between regions with very different behaviors in the magnetosphere properties are observed at $M_{p} \sim 1.0 M_{\oplus}$ and $M_{p} \sim 1.8 M_{\oplus}$ in the middle and rightmost panels of the standoff radius and polar cap area contours. Planets to the right of these boundaries still have a completely liquid core and therefore produce weaker PMFs (lower standoff radius and larger polar cap areas). On the other hand, the inner cores in planets to the left of the these boundaries have already started to grow and therefore their PMFs are stronger. 


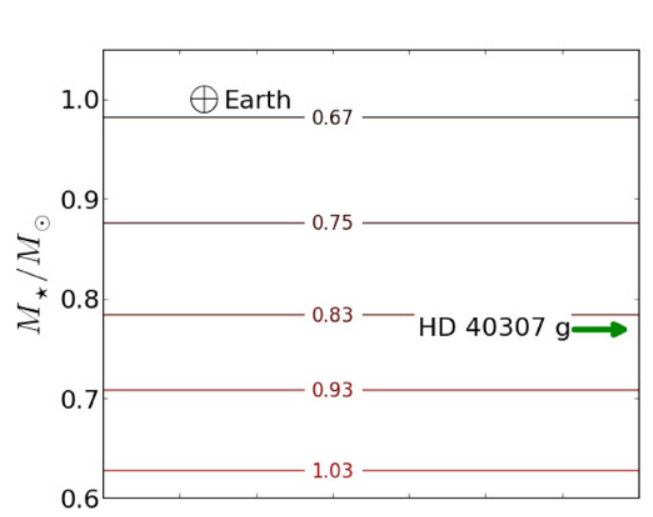

Magnetopause Field $B_{m p}(\mu \mathrm{T})$
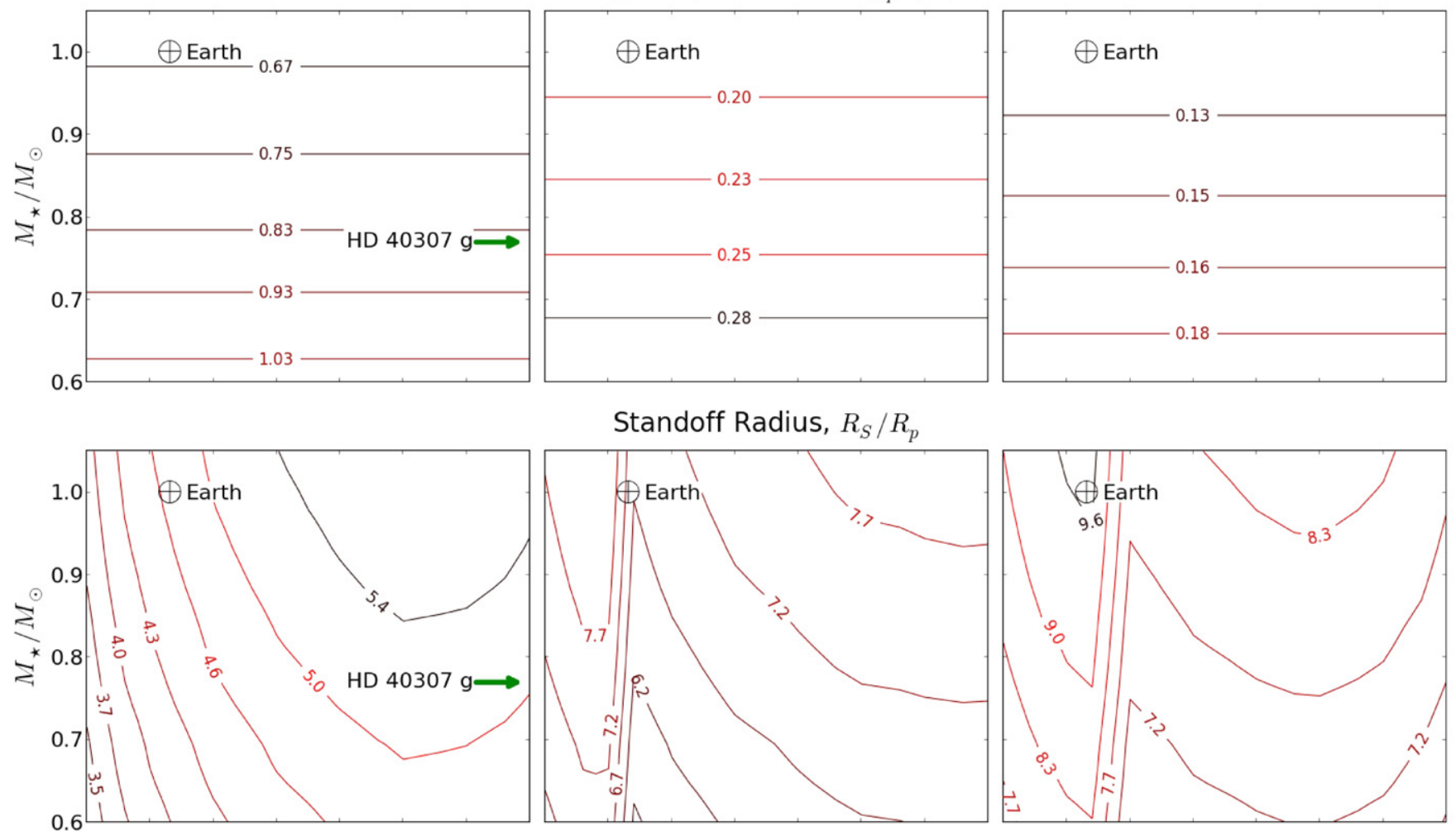

Standoff Radius, $R_{S} / R_{p}$
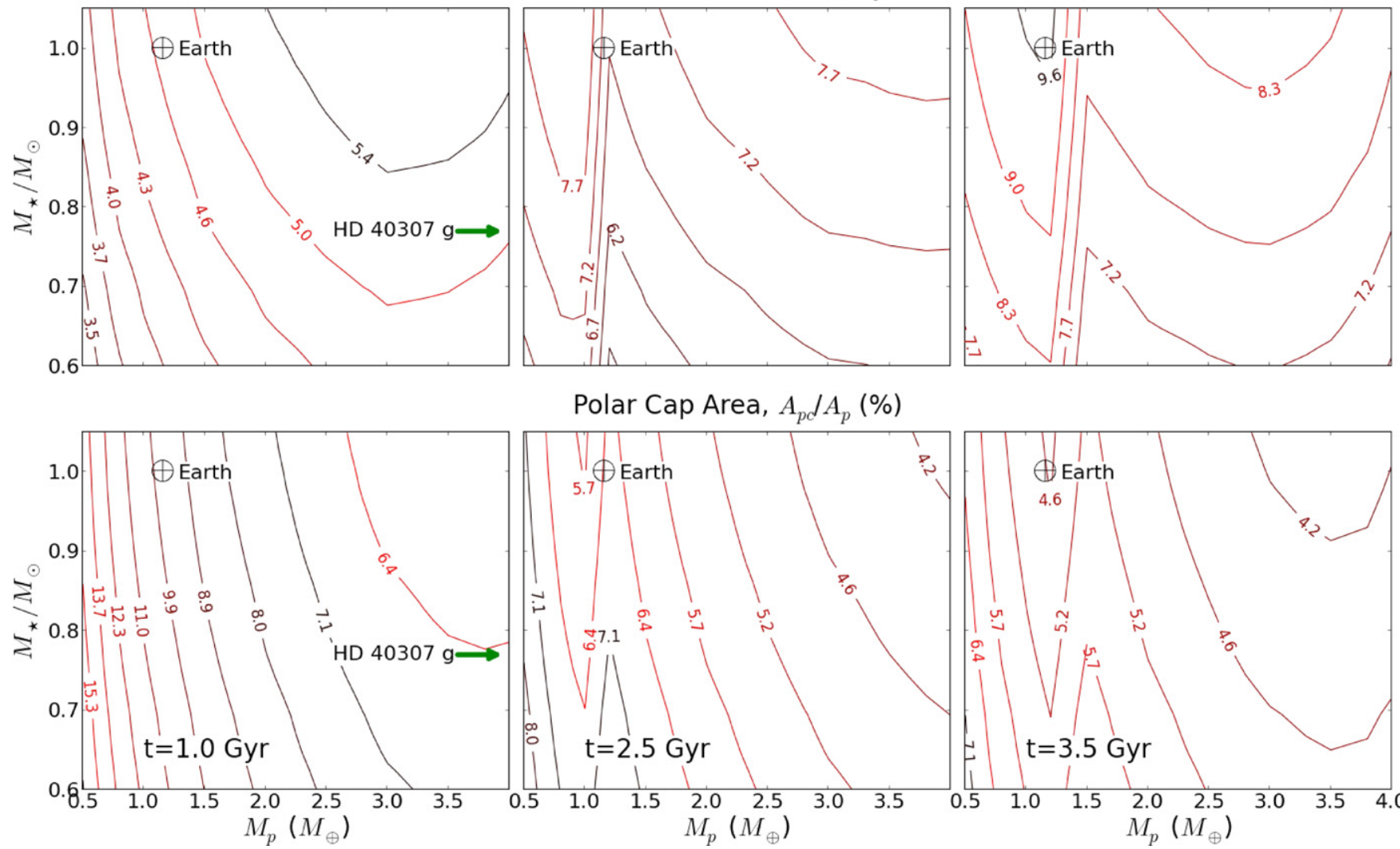

Polar Cap Area, $A_{p d} / A_{p}(\%)$

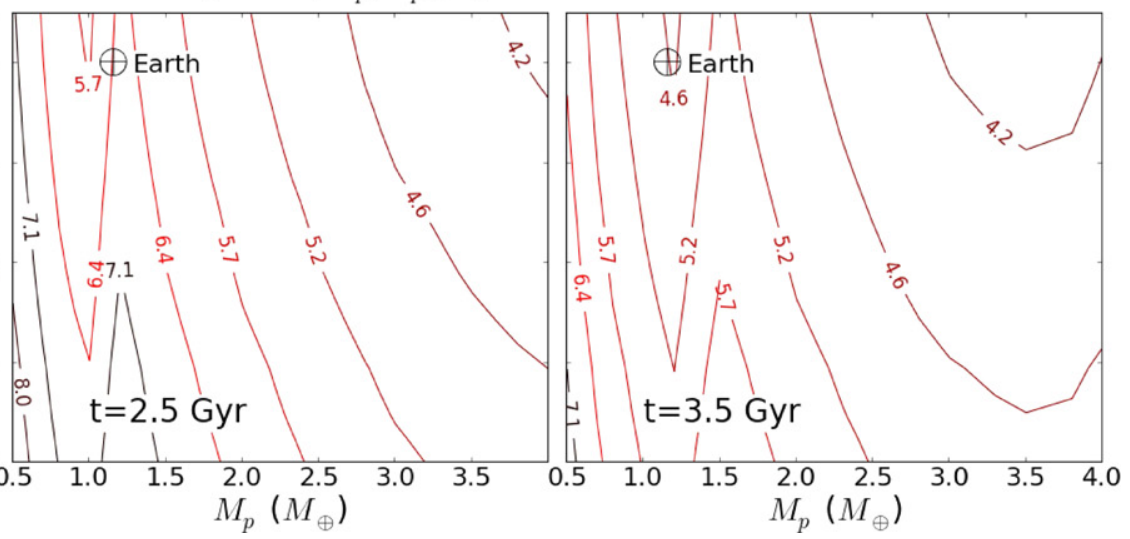

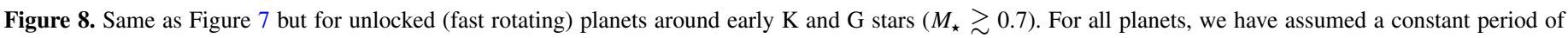
rotation $P=1$ day.

(A color version of this figure is available in the online journal.)

Unlocked planets (Figure 8) are better protected than slowly rotating tidally locked planets by developing extended magnetospheres $R_{S} \gtrsim 4 R_{p}$ and lower polar cap areas $A_{\mathrm{pc}} \lesssim 10 \%$. It is interesting to note that in both cases and at times $t \sim 1 \mathrm{Gyr}$ a smaller planetary mass implies a lower level of magnetic protection (lower standoff distances and larger polar caps). This result seems to contradict the idea that low-mass planets $\left(M_{p} \lesssim 2\right)$ are better suited to develop intense and protective PMFs (Gaidos et al. 2010; Tachinami et al. 2011; Zuluaga \& Cuartas 2012). To explain this contradiction, one should take into account that magnetic protection as defined in this work depends on dipole moment instead of surface magnetic field strength. Since dipole moment scales as $\mathcal{M} \sim B_{\mathrm{dip}} R_{p}^{3}$, more massive planets will have a better chance of having large and protective dipole moments.
It is interesting to compare the predicted values of the maximum dipole moment calculated here with the values roughly estimated in previous attempts (Grießmeier et al. 2005; Khodachenko et al. 2007; López-Morales et al. 2011). On one hand, Khodachenko et al. (2007) estimate dipole moments for tidally locked planets in the range $0.022-0.15 \mathcal{M}_{\oplus}$. These values have been systematically used in the literature to study different aspects of planetary magnetic protection (see, e.g., Lammer et al. 2010 and references therein). For the same type of planets, our model predicts maximum dipole moments almost one order of magnitude larger $\left(0.15-0.60 \mathcal{M}_{\oplus}\right)$ with the largest differences found for the most massive planets $\left(M \gtrsim 4 M_{\oplus}\right)$. These differences arise from the fact that none of the scaling laws used by Khodachenko et al. (2007) depend on the convective power. In our results, the dependency on power explains the differences 


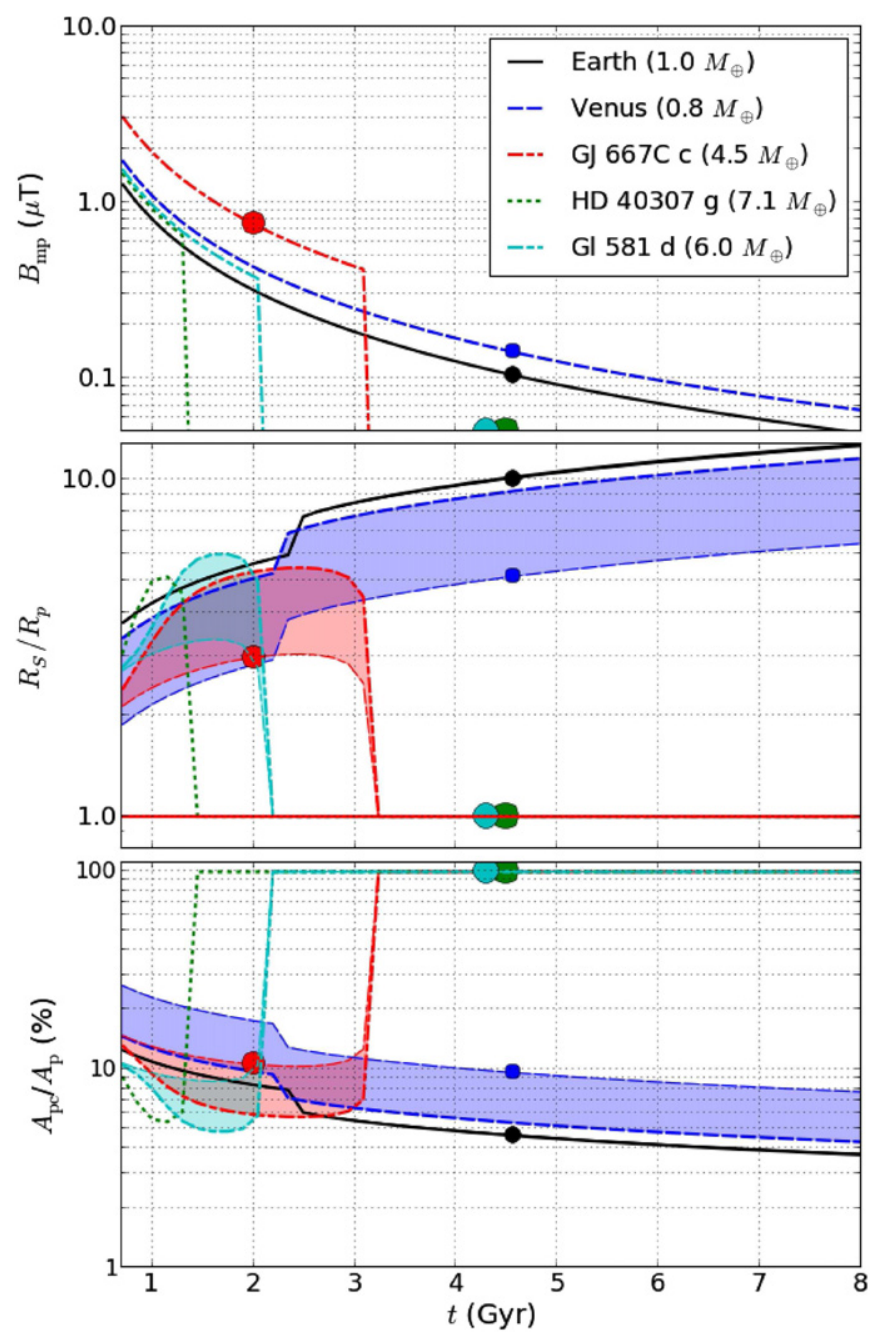

Figure 9. Evolution of magnetosphere properties for the habitable SEs already discovered, the Earth, and a "hydrated" version of Venus (low viscosity mantle and mobile lid). The shaded regions are bounded by the properties calculated at a minimum rotation period of $P \approx 1$ day (upper and lower bounds in standoff radius and polar cap area curves, respectively) and the maximum period of rotation $P \approx P_{o}$ corresponding to a perfect match between the rotation and orbital periods (tidal locking). Magnetopause fields do not depend on the rotation period of the planet. The filled circles are the predicted present day magnetosphere properties computed according to the properties summarized in Table 2.

(A color version of this figure is available in the online journal.)

between massive planets and lighter planets especially at early times. On the other hand, López-Morales et al. (2011) estimate magnetic dipolar moments of tidally locked SEs in the range 0.1-1.0 $\mathcal{M}_{\oplus}$. These values are compatible with our results. In their work, López-Morales et al. (2011) use the same powerbased scaling laws we applied here but assume a rather simple interior model and a static thermal model where the convective power is set such that maximizes the efficiency with which the convective energy is converted into the magnetic field.

A more detailed account of the evolution of magnetosphere properties for the habitable planets already discovered is presented in Figure 9. In all cases, we have assumed that all planets have compositions similar to Earth (RTEM). Although almost all planets are tidally locked, we have also computed the magnetic properties for a primordial period of rotation $P=1$ day.

The case of the "hydrated" Venus is particularly interesting in order to analyze the rest of planets. The dynamo of the actual
Venus probably shut down at $t=3 \mathrm{Gyr}$ as a consequence of the drying of the mantle (Christensen et al. 2009). A massive loss of water induced by a runaway greenhouse and insufficient early magnetic protection played a central role in the extinction of the early Venusian PMF. The evolution of the PMF in the potentially habitable planets GL 581d, GJ 667Cc, and HD 40307g could have a similar fate. Their masses are much larger and therefore their atmospheres are protected by stronger gravitational fields.

For the planet HD $40307 \mathrm{~g}$, our RTEM predicts a late shutdown of the dynamo $t_{\text {dyn }} \sim 4$ Gyr. According to our reference model, the planet is presently devoid of a dynamo-generated magnetic field. However, being around a $\mathrm{K}$ star $\left(M_{\star} \sim 0.7\right)$, the stellar wind and XUV radiation have probably decreased enough that they do not presently represent a real threat to its atmosphere.

GL 581d and GJ 667Cc are located in the HZ of dM stars where the stellar wind pressure and XUV radiation, even at times as late as $4 \mathrm{Gyr}$, are intense enough to erode their atmospheres or to make them lose their water content. The RTEM predicts that, for an Earth-composition, GL 581d at present times already lost its dynamo and has been exposed for almost 2.5 Gyr to the harmful effects of the stellar wind and CR. The planet, however, is the most massive of the three planets and probably has a thick atmosphere that is able to withstand the continuous aggression of its host star.

Given the estimated age of the GJ 667C system ( $t \approx 2$ Gyr), the RTEM predicts that the planet still has a dynamo (red circle in Figure 9). Magnetosphere properties are very close to that of our "hydrated" Venus 4 Gyr ago. However, its mass is lower than that of GL 581d and it is located at the inner edge of the $\mathrm{HZ}$ where the exposition to the XUV radiation from its host star (a young M1 star) could have been enough to induce massive loss of atmospheric gases including water. We will come back on these issues in Section 6.1 when we show how to estimate the atmospheric mass-loss rate for this particular planet.

\subsection{Toward an Estimation of the Atmospheric Mass Loss}

Combining the model of magnetosphere evolution developed here with models of thermal and non-thermal atmospheric escape, it would be possible to estimate the mass-loss rate from atmospheres of magnetized and unmagnetized potentially habitable planets. This is a fundamental goal to be pursued in the near future if we want to assess the actual habitability of TPs in the HZ of their host stars presently known and those discovered in the future. The complex interaction between an inflated atmosphere and its protective magnetosphere and large uncertainties in the surface fluxes of atmospheric gasses that compensate the loss of volatiles induced by the action of the stellar wind render this goal hard to achieve at present. Despite these limitations, we can still make order of magnitude estimations based on our own results and the mass-loss rate computed, for example, in the recent works by Tian et al. (2008), Tian (2009), and Lammer et al. (2012).

Atmospheric thermal mass loss induced by the exposition to X-rays and EUV stellar radiation (XUV) have been estimated for the case of Earth-like $\mathrm{N}_{2}$-rich atmospheres (Watson et al. 1981; Kulikov et al. 2006; Tian et al. 2008) and dry Venus-like $\mathrm{CO}_{2}$-rich atmospheres (Tian 2009; Lammer et al. 2012). One critical property of an inflated atmosphere is essential to evaluate the exposition of such atmospheres to further non-thermal processes: the radius of the exobase $R_{\text {exo }} . R_{\text {exo }}$ is defined as the distance where the mean-free path of atmospheric particles could be comparable to the size of the planet. 
When the radius of the exobase is comparable to or larger than the magnetic standoff distance $R_{S}$, we will say that the planet is unmagnetized. Under these conditions, the gases escaping from the exosphere will be picked up by the stellar wind and lost to the interplanetary space. On the other hand, if the exobase is well inside the magnetosphere (which is the case of the Earth today) atmospheric gasses escaping thermally from the exosphere could stay trapped by the magnetic field forming a plasmasphere. Planets under this condition will be magnetically protected and the mass-loss rate is expected to be much lower than for unmagnetized planets.

Using the conservative estimation of the X and EUV luminosities of main-sequence stars given by Garcés et al. (2011), we have estimated the XUV flux at the top of the atmospheres of GL 581d, GJ 667Cc, and HD 40307g during the first critical gigayear of planetary evolution. The planet that received the minimum amount of XUV radiation is HD $40307 \mathrm{~g}$ with $F_{\mathrm{XUV}}=10-35$ PEL $\left(1 \mathrm{PEL}=0.64 \mathrm{erg} \mathrm{cm}^{-2} \mathrm{~s}^{-1}\right.$ is the present Earth value; Judge et al. 2003; Guinan et al. 2009). GL 581d was exposed in the first gigayear to a flux of $F_{\mathrm{XUV}}=150-250 \mathrm{PEL}$ while GJ $667 \mathrm{Cc}$ received the maximum amount of XUV radiation among them, $F_{\mathrm{XUV}}=450-800$ PEL.

Using the recent results by Tian (2009) that computed the exosphere properties of massive SEs, i.e., $M_{p} \geqslant 6 M_{\oplus}$, subject to different XUV fluxes, we can estimate the exosphere radius and mass-loss rate for our three habitable SEs. Actually, since the Tian (2009) results are only available for planets with a minimum mass of $M_{p}=6 M_{\oplus}$, a qualitative extrapolation of the results for 10,7 , and $6 M_{\oplus}$ (see Figures 4 and 6 in his paper) shows that exobase radius and mass-loss rates are larger for less massive planets. This is particularly useful for trying to apply the Tian's results to GJ 667Cc $M_{p} \approx 4.5 M_{\oplus}$ and other less massive potentially habitable planets. In these cases, we will use the results by Tian (2009) to calculate a lower bound of the exobase radius and mass-loss rates.

Using the estimated XUV flux for HD 40307g $\left(M_{p} \approx 7 M_{\oplus}\right)$ and assuming an initial $\mathrm{CO}_{2}$-rich atmosphere, Tian's results predict that the exosphere of the planet and hence its mass-loss rate was low enough to avoid a significant early erosion of its atmosphere (see Figures 4 and 6 in his paper). This is true at least during the first 1-2 Gyr during which our magnetic protection model predicts that the planet was enshrouded by a protective magnetosphere (see Figure 9). After dynamo shutdown, the atmosphere of HD 40307g has been exposed to the direct action of the stellar wind. Assuming a stellar age of $4.5 \mathrm{Gyr}$ (Tuomi et al. 2012), this effect has eroded the atmosphere for 3-4 Gyr. During this unmagnetized phase, the atmospheric mass-loss rate can be simply estimated as $\dot{M} \approx \alpha m n v_{\text {eff }}$ (Zendejas et al. 2010) where $\alpha$ is the so-called entrainment efficiency and $m, n$, and $v_{\text {eff }}$ are the mass, number density, and effective velocity of the stellar wind as measured at planetary distance (see Equation (2)). Using a entrainment efficiency $\alpha \sim 0.3$ (which is appropriate, for example, to describe the mass loss of the Venus atmosphere), we find that the total mass loss during the unmagnetized phase is less than $1 \%$ of a conservative estimate of the total volatile content of the planet (Tian 2009). Although our model provides only upper limits to magnetic protection and the planet could have, for example, a lighter nitrogen-rich atmosphere which is more prone to XUV-induced mass losses (Watson et al. 1981; Kulikov et al. 2006; Tian et al. 2008), this preliminary estimation suggests that HD 40307g probably still preserves a dense enough atmosphere that is able to sustain surface liquid water and hence to be actually habitable.
The case of GL 581d $\left(M_{p} \approx 6 M_{\oplus}\right)$ is quite different. Assuming that our estimations of the XUV flux are correct, the exosphere radius predicted by Tian (2009) should be close to the actual one. In this case at times $t \sim 1 \mathrm{Gyr}, R_{\text {exo }}=1.8-2.3 R_{p}$. However, our reference magnetosphere model predicts for this planet magnetic standoff distances $R_{S}>2.7$ at all times. We conclude that by using our estimations GL 581d could have been protected by its intrinsic magnetic field during the critical early phases of planetary evolution and probably has preserved the critical volatiles in its atmosphere. Still, the uncertainties in the exosphere model or in the atmospheric composition and of course in the magnetic model developed here should lead to a different conclusion and further theoretical and probably observational investigation are required.

The most interesting case is that of GJ $667 \mathrm{Cc}\left(M_{p} \approx 4.5 M_{\oplus}\right)$. The minimum exosphere radius predicted for this planet at $t \sim 1$ Gyr lies between 3.0 and $4.5 R_{p}$ while, according to our magnetosphere model, the magnetic standoff distance is $R_{S}<3 R_{p}$ up to $2 \mathrm{Gyr}$. Since the exosphere radius should actually be larger than that predicted with the Tian (2009) model and our magnetic model is actually optimistic, the chances that this planet was unprotected by its magnetic field in the critical first gigayear are high. But exposition does not necessarily mean a complete obliteration of the atmosphere (see, for example, the case of Venus). To evaluate the level of thermal and nonthermal obliteration of the atmosphere, we need to estimate the actual mass-loss rate. At the XUV fluxes estimated at the top of the atmosphere of this planet during the first gigayear, the minimum thermal mass-loss rate of carbon atoms from a $\mathrm{CO}_{2}$ rich atmosphere will be larger than $2-4 \times 10^{10}$ atoms cm $\mathrm{cm}^{-2} \mathrm{~s}^{-1}$ (see Figure 6 in Tian 2009). We should recall that this is actually the value for a $6 M_{\oplus} \mathrm{SE}$. For the actual mass of the planet, 4.5 $M_{\oplus}$, the mass-loss rate could be even larger. Moreover, if, as predicted here, the exosphere is directly exposed to the stellar wind, non-thermal processes can contribute to a larger increase in the mass loss from the planetary atmosphere. At the minimum mass-loss rate, the exposed GJ 667Cc atmosphere could have lost more than $\sim 10^{46}$ atoms of carbon in just $\sim 100 \mathrm{Myr}$ and in the first gigayear the amount of carbon thermally lost to space could rise to $\sim 10^{47}$ atoms. If we scale up linearly with planetary mass, the total inventory of $\mathrm{CO}_{2}$ in the atmosphere, crust, and mantle of Venus, which is $2-3 \times 10^{46}$ molecules (see Tian 2009 and references therein), a $4.5 M_{\oplus}$ planet will have a total budget of $\sim 10^{47} \mathrm{CO}_{2}$ molecules. In summary, at the minimum mass-loss rate and assuming a relatively rapid degassing of the planet, Gj 667Cc could have lost its total inventory of carbon to interplanetary space in the first couple of gigayears. Even assuming that large amounts of $\mathrm{CO}_{2}$ are still trapped in the mantle and crust of the planet, its atmosphere should be rapidly obliterated by the stellar wind. We speculate that GJ 667Cc is a sort of "Venus-like" planet. Regardless the fact that the planet is inside the radiative $\mathrm{HZ}$, it has lost its capacity to support life via a massive stellar-wind-induced loss of volatiles.

\section{DISCUSSION}

Applying simplified thermal evolution model and dynamo scaling laws to planets whose bulk properties are barely known or even hypothetical, it is challenging and probably raises more questions than it attempts to solve. Further observations of the potentially habitable planets should be required to present their precise physical properties and to reliably model its interior and thermal evolution. Moreover, continued observational efforts 
to look for direct evidence or proxies of planetary magnetospheres and any other signatures of magnetic protection, though challenging, should also be attempted. Here, we discuss the assumptions on which our global model relies, its uncertainties as measured by the sensitivity of the model to changes in key physical parameters, and the missing pieces of information and present observational limitations to confirm or improve this and other models of planetary magnetic protection.

\subsection{Model Assumptions}

The strength of a physical model depends on the hypothesis and assumptions on which it relies. Apart from numerous albeit very common assumptions, the magnetic protection model presented here depends on three major assumptions which we discuss in the following paragraphs.

We have assumed that TPs always develop an initially metallic liquid core, irrespective of their composition and early formation history. This is not necessarily true. The formation of a metallic liquid core would depend on very complex processes and other barely known physical factors. It has been shown, for example, that under extreme water oxidation of iron the formation of a metallic core will be avoided (Elkins-Tanton \& Seager 2008). In this case, silicate coreless planets will be formed. On the other hand, even if a planet is well differentiated the core could be solid from the beginning (see, e.g., VAL06). However, it should be emphasized here that our model provides only the best-case scenario of magnetic protection. Therefore, if, under the assumption of having a liquid metallic core, a planet is found to be lacking enough magnetic protection, the case when the planet is not well differentiated or when it never develops a liquid core will be even worse. In these cases, the conclusions drawn from our model will be unchanged.

The calculation of key magnetosphere properties relies on very simplistic assumptions about the complex physics behind the interaction between planetary and interplanetary magnetic fields and stellar wind. In particular, standoff distances calculated with Equation (5) assume a negligible plasma pressure inside the magnetosphere. This condition could be violated in planets with very inflated atmospheres and/or at close-in orbits. Under these extreme conditions, the magnetic standoff distance given by Equation (5) could be a poor underestimation of the actual magnetopause distance. However, the weak dependence of standoff distances and polar cap areas of the stellar wind dynamic pressure offers some idea as to the actual role that magnetospheric plasma pressure may pay in determining the size of the magnetosphere. Adding a plasma pressure term $P_{\mathrm{pl}}$ to the magnetic pressure $P_{\mathrm{mp}}$ in the left-hand side of Equation (3) is equivalent to subtracting it from the stellar wind dynamic pressure $P_{\mathrm{sw}}$ in the right-hand side. An effective stellar wind pressure $P_{\mathrm{sw}}^{\prime}=P_{\mathrm{sw}}\left(1-P_{\mathrm{pl}} / P_{\mathrm{sw}}\right)$ would replace the stellar wind term in the standoff distance definition (Equation (5)). As a result a plasma pressure correcting factor $\left(1-P_{\mathrm{pl}} / P_{\mathrm{sw}}\right)^{-1 / 6}$ will modify our estimated purely magnetic standoff distance. Even in a case where the plasma was able to exert a pressure $50 \%$ of that of the stellar wind, the standoff distance will increase by only $10 \%$. On the other hand, in order to have a standoff distance one order of magnitude larger than that estimated using Equation (5), the plasma pressure inside the magnetosphere should amount to $99.999 \%$ of the total pressure. This is precisely what an unmagnetized planet would look like. In summary, including more realistic condition in the definition of the magnetosphere boundary will not greatly modify our results.
A final but no less important assumption in our model is that of quiet stellar wind conditions. We have only taken into account average or quiet stellar wind conditions. We have completely neglected the effects of large but transient conditions such as those produced during coronal mass ejections (CMEs). To model the effect that a steady flux/influx of CME plasma could have on close-in planets, we can modify the stellar wind pressure by maintaining the nominal velocity of the plasma but increasing the number density of wind particles by a factor of two. ${ }^{4}$ Taking into account that $R_{S} \sim P_{\mathrm{SW}}^{-1 / 6}$, we found that under the harsher conditions the magnetosphere radius and polar cap areas will be modified only by $10 \%-30 \%$ with respect to the nominal or quiet values computed here. This simplified estimation shows that our results seem to be robust in relation to uncertainties in the stellar wind pressure. However, given the complexity of the interaction between the magnetosphere and the stellar wind under active phases, a further examination of this case is required and is left to future research.

\subsection{Sensitivity Analysis}

In order to study the effect that uncertainties in several critical thermal evolution parameters have in the prediction of the overall magnetic protection of potentially habitable TPs, we performed a sensitivity analysis of our model. For this purpose, we independently varied the value of six carefully chosen parameters of the model (see below) and compared the predicted dipole moment, the time of inner core formation, and the dynamo lifetime with the same values obtained using the RTEM.

We performed these comparisons for planets with five different masses: $0.7,1.0,3.5,4.5$, and $6.0 M_{\oplus}$ (see Figure 10). These masses correspond approximately with those of the habitable planets already discovered (see Table 2) including a hydrated Venus and present Earth. In all cases, for simplicity we assume a primordial period of rotation of $P=1$ day.

Since the dipole moment is an evolving quantity, in Figure 10 we plotted the average value of this quantity as calculated in the interval 0.7-2.0 Gyr. For times earlier than $0.7 \mathrm{Gyr}$, the stellar wind pressure is uncertain and the magnetic protection cannot be estimated (as discussed in Section 5.2, observations suggest lower stellar wind pressures at times earlier than this). For times larger than $2.0 \mathrm{Gyr}$, the flux of XUV radiation and the stellar wind pressure have decreased below the initial high levels. Although an average of the dipole moment is not phenomenologically relevant, it could be used as a proxy of the overall magnetic shielding of the planet during the harsh early phases of stellar and planetary evolution.

After studying the full set of physical parameters involved in our interior structure and thermal evolution models (see Table 1), we identified six critical parameters whose values could have noticeable effects on the results or are subject to large uncertainties. We performed an analysis of the sensitivity that the model has to the variation of the following physical parameters:

1. The core mass fraction, $C M F$. This is the fraction of the planetary mass represented by the metallic core. This parameter is determined by the $\mathrm{Fe} / \mathrm{Si}$ ratio of the planet that it is fixed at planetary formation or could be altered by exogenous processes (e.g., late large planetary impacts).

\footnotetext{
4 Under typical conditions of solar CME, the velocity of the wind is not greatly modified but the plasma densities increase by up to 5-6 times over the average particle density.
} 

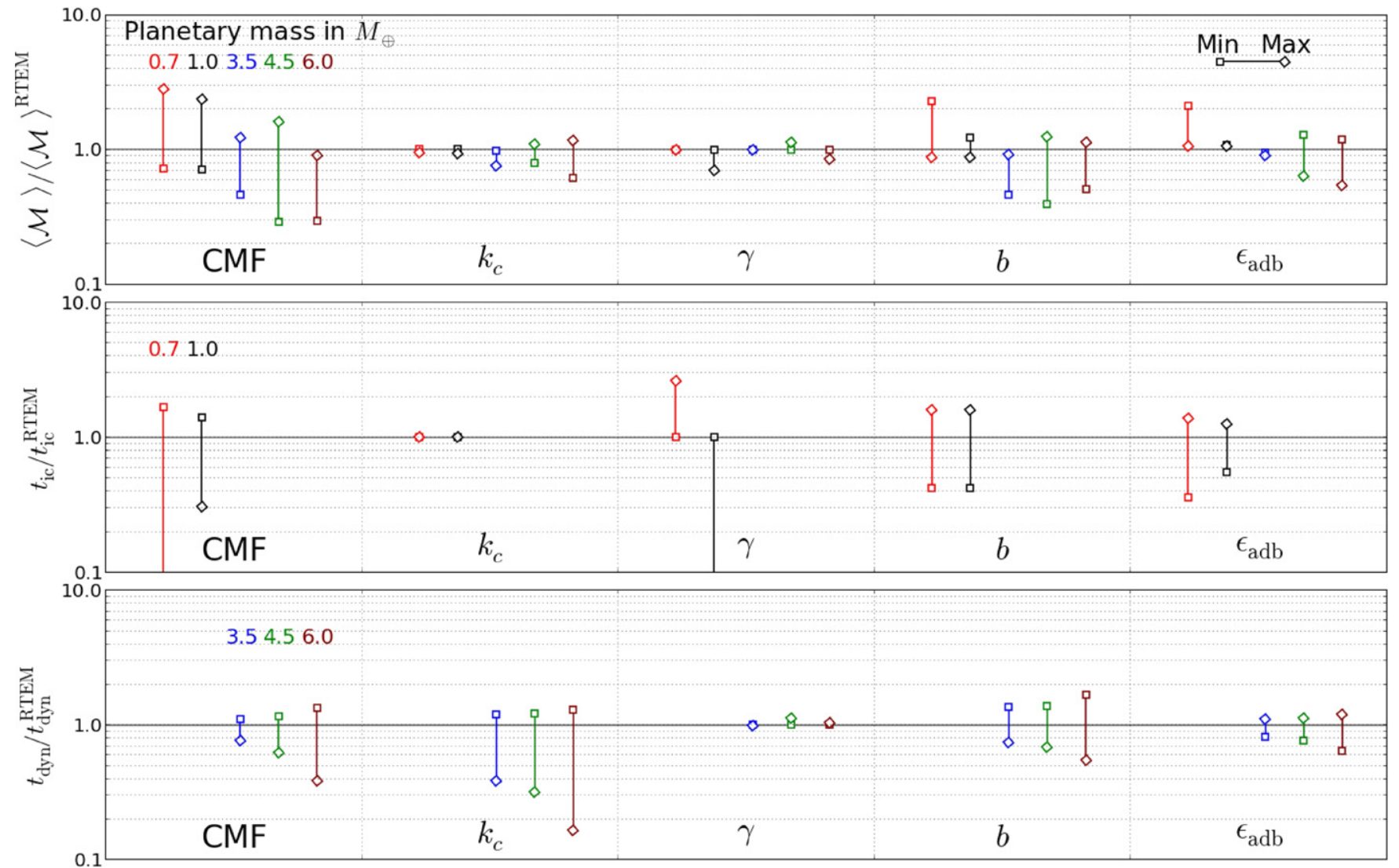

Figure 10. Sensitivity analysis of our reference thermal evolution model (RTEM). The squares and diamonds indicate the relative values of three critical magnetic properties, $\left\langle\mathcal{M}_{\text {dip }}\right\rangle$ (the average of the dipole moment between 0.7 and $2 \mathrm{Gyr}$ ), $t_{\text {ic }}$ (time of inner core formation), and $t_{\text {dyn }}$ (dynamo lifetime), as calculated by the thermal evolution model. For the analysis, five different key thermal evolution parameters were independently changed with respect to the reference value in the RTEM: the core mass fraction (CMF), the thermal conductivity of the core $\left(k_{c}\right)$, the Grüneisen parameter at core conditions $\left(\gamma_{0 c}\right)$, the high pressure viscosity rate coefficient $(b)$, and the adiabatic factor $\left(\epsilon_{\text {adb }}\right)$. The results obtained when the minimum value of the parameters were used are indicated with squares. Conversely, the results obtained with the maximum value of each parameter are indicated with diamonds.

(A color version of this figure is available in the online journal.)

Our reference model uses Earth's value CMF $=0.325$, i.e., assumes that all planets are dominated by a silicaterich mantle. As a comparison, Mars has a $\mathrm{CMF}=0.23$ and the value for Mercury is CMF $=0.65$ (it should be noted that Mercury could have lost a significant fraction of its mantle silicates increasing the total iron fraction, probably after an early large impact). The CMF determines the size of the core and hence the thermal properties of the convective shell where the magnetic field is generated. For our sensitivity analysis, we have taken two extreme values of this parameter, $\mathrm{CMF}=0.23$ (a Mars-like core) and $\mathrm{CMF}=0.43$ (an iron-rich core). Planets with larger cores have low pressure olivine mantles and our rheological model becomes unreliable.

2. The initial temperature contrast at the $C M B, \Delta T_{\mathrm{CMB}}=$ $\epsilon_{\mathrm{adb}} \Delta T_{\mathrm{adb}}$ (see Equation (25)). This is one of the most uncertain properties in thermal evolution models. The initial core temperatures would be determined by random processes involved in the assembly and differentiation of the planet. It could vary widely from planet to planet. In order to fit the thermal history of the Earth (time of inner core formation, present size of the inner core, and magnetic field strength), we set $\epsilon_{\mathrm{adb}}=0.7$ and applied the same value to all planetary masses. In our sensitivity analysis, we varied this parameter between two extreme values of 0.6 and 0.8 . Though we are not sure that this interval is representative of planets with very different masses and formation histories, our analysis at least provides the magnitude and sign of the effect that this parameter has on the dynamo properties predicted by our thermal evolution model.

3. High pressure viscosity rate coefficient, $b$ (see Equation (26)). Rheological properties of silicates inside the mantle are among the most uncertain aspects of thermal evolution models. They critically determine, among other key quantities, the amount of heat that the core and mantle could transport through their respective boundary layers (see Equations (13), (20), and (22)). We found that the viscosity of the lower mantle (perovskite) is the most important source of uncertainties in our thermal evolution model. The formula used to compute viscosity at that layer (see Equation (27)) strongly depends on temperature and pressure and the parameter controlling this dependence is the "rate coefficient" $b$. In the RTEM, we used the value $b=12.3301$ to reconstruct the thermal properties of the Earth. Using this value, all the figures reflect the strong sensitivity of the model to this parameter. To study the impact of $b$ in the model results, we varied it in the interval 10-14.

4. Iron thermal conductivity, $k_{c}$. This parameter controls the amount of heat coming from the core. In the RTEM, we used a value $k_{c}=40 \mathrm{~W} \mathrm{~m}^{-1} \mathrm{~K}^{-1}$ that fits the thermal evolution history and present magnetic field of the Earth (see Table 1). Although recent first-principles analysis 
suggests that values as large as $150-250 \mathrm{~W} \mathrm{~m}^{-1} \mathrm{~K}^{-1}$ could be common at Earth's core conditions (Pozzo et al. 2012), we conform here to the standard values of this parameter. Further investigations to explore values as large as those found by Pozzo et al. (2012) should be attempted. For our sensitivity analysis, we varied $k_{c}$ between 35 and $70 \mathrm{~W} \mathrm{~m}^{-1} \mathrm{~K}^{-1}$, two values which are inside the typical uncertainty assumed for this property.

5. Grüneisen parameter for iron, $\gamma_{0 c}$. This is one of the most critical parameters of the equation of state, especially at core conditions. It strongly affects the mechanical structure, temperature profile, and phase of iron in the metallic core (for a detailed discussion on the sensitivity of interior structure models to this parameter, see, e.g., VAL06). In the RTEM, we used the reference value $\gamma_{0 c}=1.36$ that fits the thermal evolution history and present magnetic field of the Earth (see Table 1). Assuming different kinds of core alloys, a relatively large range of values of this parameter have been used in literature (see VAL06 and references therein). The Grüneisen parameter values have been found in the range of 1.36-2.338. Since our RTEM value is at the lower end of this range, for our sensitivity analysis we have recalculated the model for a larger value of 2.06.

Other uncertain parameters, such as the critical Rayleigh number at the CMB, $\mathrm{Ra}_{c}$, which is also varied to study the sensitivity of thermal evolution models (E. Gaidos 2011, private communication), were also studied. We did not find significant sensitivity of our model to the variation of those parameters.

In Figure 10 we depict the relative variation in the aforementioned magnetosphere and dynamo properties when each of the previously described parameters were varied independently.

We found that planetary composition (CMF) and mantle viscosity are responsible for the largest uncertainties in the predicted magnetic properties of the planet. Planets with small metallic cores have on average low magnetic dipole moments (squares in the first column of the upper panel). This is mainly due to a geometrical effect. The total heat produced by the core and hence the magnetic field strength at the core surface is of the same order as Fe-poor and Fe-rich planets. However, a small core also means a lower magnetic dipole moment, i.e., $\mathcal{M} \sim R_{c}^{3}$. Planets with lower iron contents also have small and hot cores and therefore the solid inner core formation and shutting down the dynamo are slightly delayed (squares in the middle and lowest panel of Figure 10).

Viscosity dependence on pressure and temperature, as quantified by the parameter $b$, has the opposite effect on planetary magnetic properties than CMF at least for EPs. A low viscosity, lower mantle will favor the extraction of heat from the metallic core increasing the available convective energy for dynamo action. On the other hand, a viscous lower mantle will delay the formation of a solid inner core and extend the lifetime of the dynamo (middle and lowest panel in Figure 10).

The effect of the Grüneisen parameter at core conditions is negligible, at least in respect to the magnetic field strength and lifetime (upper and lower panels) which are the most critical properties affecting planetary magnetic protection. Only the time of the inner core formation is strongly affected by changes in this parameter. In planets smaller than Earth, inner core solidification can be delayed up to three times the reference value. On the other hand, with a larger Grüneisen parameter, the inner cores of EPs could become solid very early in their thermal histories, even almost from the beginning. This is a result of the interplay between the resulting evolution of the thermal profile and the solidus.

The effect of the initial temperature contrast across the CMB, quantified by the parameter $\epsilon_{\mathrm{adb}}$, goes in the same direction as viscosity. The reasons for this behavior are, however, far more complex. A larger initial temperature contrast across the CMB also implies a larger initial temperature at the core center. Although a hotter core also produces a larger amount of available convective energy, the time required for iron to reach the solidification temperature is also larger. The dynamo of planets with $M_{p}<2 M_{\oplus}$ and hot cores (large $\epsilon_{\mathrm{adb}}$ ) is weaker than that of more massive planets during the critical first couple of gigayears where the average is calculated. Planets with colder cores develop solid inner cores almost from the beginning and the release of latent and gravitational energy feeds stronger dynamos.

The case of more massive planets, $M_{p}>2 M_{\oplus}$, where the condition for an inner core formation is never reached during the dynamo lifetime, is different. In this case, planets with hot cores (large lower mantle viscosities or high temperature contrasts along the $\mathrm{CMB}$ ) produce large amounts of available convective energy. A larger convective power will produce a larger value of the local Rossby number. Thus, massive planets with hot cores also have multipolar dynamos and hence lower dipole magnetic moments and a reduced magnetic protection.

Thermal conductivity affects the results of the thermal evolution model less. Besides the case of massive planets where differences on the order of $10 \%-30 \%$ in the magnetic properties are observed when $k_{c}$ varies between its extremes, the magnetic properties calculated with our RTEM seem very robust in comparison with variations of these two properties. However, it should be mentioned that this result applies only when a standard value of $k_{c}$ is assumed. Further investigations to explore the recent findings (Pozzo et al. 2012) concerning the possibility that $k_{c}$ could be larger by factors as large as $2-3$ should be attempted.

In summary, despite the existence of a natural sensitivity of our simplified thermal evolution model to uncertainties on their free parameters, the results presented in this paper seem to be correct at least on the order of magnitude. Moreover, since the standoff distance and polar cap areas, which are the actual proxies to magnetic protection, goes as $\mathcal{M}^{1 / 3}$, a one order of magnitude estimation of $\mathcal{M}$ will give us an estimation of the level of magnetic protection by a factor of around two.

To clarify this point, let us consider the case of GL 581d. If we assume, for example, that its iron content is much less than that of the Earth (as was assumed in the RTEM model), but the rest of critical thermal properties are essentially the same, the average standoff distance (polar cap area) at the critical first gigayear will be off by only $\sim 30 \%$ with respect to the prediction depicted in Figure 9. More interesting is to note that the uncertainties due to the unknown period of rotation (shaded area in Figure 9) seem to be much larger than those coming from the uncertainties in the thermal evolution model.

\subsection{Observational Support}

Validating or improving thermal evolution models and dynamo scaling laws for the case of SEs presently represents a huge observational challenge. The available sensitivity of our best instruments on Earth and in space is rather insufficient. New and/or improved instruments and observational techniques will be required to detect, catalog, and compare the thermal and magnetic properties of low-mass planets in 
the future. However, the importance that the detection and characterization of the magnetic properties of potentially habitable planets discovered in the future in order to assess their true habitability clearly justifies the effort.

The first goal seems to be the direct or indirect detection of SE magnetospheres. Four methods, some of which are already used in our own solar system, could be devised to achieve this goal: (1) the detection of radio waves coming from synchrotron and cyclotron radiation produced by plasma trapped in the magnetosphere, (2) the detection of a bow shock or a tail of ions produced by the interaction of the planetary atmosphere and magnetosphere with the stellar wind or the interplanetary plasma, (3) the detection of planetary auroras, and (4) spectroscopic observations of a non-equilibrium atmospheric chemistry induced by a high flux of CR (this is actually a negative detection of a magnetosphere).

The first (radio emission) and second methods (bow shock or tail) have already been studied in detail (Bastian et al. 2000; Farrell et al. 2004; Grießmeier et al. 2007b; Lazio et al. 2009; Vidotto et al. 2010, 2011). Its reliability, at least for the case of planets with intense magnetic fields or placed very close to their host star, has been already tested.

If synchrotron or cyclotron radiation coming from the magnetosphere of TPs could be detected, the power and spectra of the radiation could be used to measure the magnetic field strength. However, even with the most sensitive instruments, e.g., the Low Frequency Array or the Long Wavelength Array, the expected power and spectra are several orders of magnitude below the threshold of detection. Powers as large as $10^{3}-10^{5}$ times the Jupiter radio emission and frequencies in the range of tens of $\mathrm{MHz}$ are required for the present detection of synchrotron radio emission in planetary magnetospheres (see, e.g., Grießmeier et al. 2007b). The magnetic field intensities and expected frequencies produced in SEs magnetospheres are several orders of magnitude lower than these thresholds and are not likely to be detected in the near future.

It has been shown recently that measurements of the asymmetry in the ingress and egress of transiting planets can be used to detect the presence of a bow shock or a tail of plasma around the planet. Vidotto et al. $(2010,2011)$ used this phenomenon to constrain the magnetic properties of Wasp-12b. The formation of a detectable bow shock depends, among other factors, on the relative velocity between the planet and the shocked plasma. Close-in planets with strong enough magnetic fields (this is precisely the case of Wasp-12b) can easily develop UV-opaque bow shocks and allow reliable detection. However, low-mass planets with relatively weak magnetic fields, such as those predicted with our models, hardly produce a detectable bow shock. It has been estimated that magnetopause fields in the range of several gauss (among other important factors such as large enough projected magnetospheric size) should be required to have a detectable signal of a bow shock (A. A. Vidotto 2012, private communication). Our habitable SEs have magnetopause fields of the order of a few microgauss (see Figure 7). The case of an ion tail coming from a weakly magnetized planet has received less attention and probably could offer better chances for a future indirect detection of the magnetic environment of low-mass planets.

Finally, the detection of far-UV (FUV) or X-ray emission from planetary auroras can also be used as a tool to directly and indirectly study planetary magnetospheres. Planetary auroras with intensities as high as $10^{2}-10^{3}$ times larger than that of the Earth are expected in close-in giant planets subject to the effects of CMEs from its host star (Cohen et al. 2011). If we estimate that a typical Earth aurora has an intensity of $1 \mathrm{kR}$ (Neudegg et al. 2001; being $1 R \sim 10^{-11}$ photons $\mathrm{m}^{-2} \mathrm{~s}^{-1} \mathrm{sr}^{-1}$ ) and assuming that $10 \%$ of a close-in Jupiter-like planet is covered by auroras producing FUV photons around $130 \mathrm{~nm}$, the total emitted power from these planets will be $\sim 10^{13} \mathrm{~W}$. If we assume that this is the present threshold for exoplanetary aurora detection, even under strong stellar wind conditions and distances typical of the HZ, the total FUV power produced by auroras in the polar cap of EPs could be only $10^{5} \mathrm{~W}$, which is eight orders of magnitude less than the present detection threshold. In addition, the FUV radiation should be detected against an intense UV background likely coming from a young and active low-mass star. If we can find ways to overpass these difficulties, the observation of the FUV and X-ray emission and its variability from auroras in potentially habitable SEs could be used as powerful probes of the magnetic environment around the planet.

\section{SUMMARY AND CONCLUSIONS}

We studied the influence that the thermal evolution of potentially habitable TPs has in the protection that an evolving planetary magnetosphere could provide against the atmospheric erosion caused by the stellar wind.

We developed a simple parameterized thermal evolution model able to reproduce the global thermal history and magnetic properties of the past and present Earth. We applied this model to predict the thermal histories of planets with masses in the range of 0.5-6.0 $M_{\oplus}$ and with chemical compositions similar to Earth. Using these results and applying up-to-date dynamo scaling laws, we predicted the magnetic properties of TPs in the HZ as a function of time, planetary mass, and rotation rate. A simple model of the evolution and interaction of the stellar wind with the PMF, which has been adapted from previous works, allowed us to compute the global properties of the magnetosphere in order to assess the level of magnetic protection that potentially habitable EPs could actually have.

We applied our model to the case of potentially habitable TPs already known (GL 581d, HD 40307g, and GJ 667Cc), to the Earth itself, and to the case of a hydrated Venus. In the case of the Earth, our model reproduces fairly well the early and present thermal and magnetic properties of our planet. In the case of the hydrated Venus, the model predicts low values the standoff distance and large polar cap areas in the first critical gigayear of planetary and thermal evolution, which are compatible with the idea that the planet lacked magnetic protection strong enough to avoid a massive loss of water and volatiles that finally lead to the shutdown of its dynamo 3 Gyr ago.

Compelling results were found in the case of the three extrasolar system potentially habitable planets already discovered. Assuming an Earth-like composition and thermal evolution parameters similar to those used in the case of the Earth (RTEM), our model predicted that the dynamos of GL 581d and HD 40307g have been already shut down. A younger GJ 667Cc seems to still have an active dynamo.

A non-trivial dependence of the magnetic properties on planetary age, planetary mass, and period of rotation has been found in general for terrestrial planets inside the $\mathrm{HZ}$ of their host stars. Thermal evolution is responsible for the non-trivial relationship among all these properties. Contrary to that found in previous works, tidally locked planets could develop relatively intense magnetic fields and extended magnetospheres. However, they also have extended polar caps and likely multipolar magnetic fields where field lines open to the interplanetary space 
and magnetotail regions, likely increasing the non-thermal mass losses.

Using recent results for the relationship between the exposition to XUV radiation, the exobase radius, and mass-loss rate from massive SEs, we estimated the level of exposure and mass losses for the three potentially habitable SEs already discovered. With the information available little could be said about the magnetic protection of HD $40307 \mathrm{~g}$. Further theoretical investigations are required to evaluate this case. Our model predicts a magnetosphere large enough to protect GL 581d against the erosive action of the stellar wind during the first critical phases of planetary evolution. However, since our model is still optimistic, further theoretical and observational analyses should be performed to establish the magnetic protection of this planet on a more solid basis. Our upper limit to the standoff distance and the most optimistic estimation of exobase radius and massloss rate from the atmosphere of GJ $667 \mathrm{Cc}$ point out the fact that this planet has already lost a large fraction of its inventory of volatiles. All the evidence compiled in this work makes GJ 667Cc a sort of "Venus analog." Although further theoretical analyses are required, our best guess is that, despite the fact that it is inside the radiative $\mathrm{HZ}$ of its host star, the planet is presently uninhabitable.

Last, but not least, we tested the robustness of our conclusions by changing several of the most sensitive input parameters of our thermal evolution model. We found that even under the present uncertainties the predicted properties of planetary magnetospheres are rather robust. We calculated that introducing large variations in the composition of the planets and the rheological and thermal properties of their interiors with respect to the RTEM, the critical magnetic properties, such as the standoff radius and the area of the polar cap, change only by a factor of two. The results are also robust in comparison with uncertainties in the stellar wind properties that could be very important in the case of close-in habitable planets around active and young dM stars.

The problem of evaluating the magnetic protection of potentially habitable planets is far from being settled. Other sources of intrinsic magnetic fields, thermal evolution, and interior structure of planets with "exotic" compositions, improved theoretical models, and new experimental evidence of the behavior of iron at high pressures and temperatures, improved and validated models of the evolution and spatial structure of stellar winds and of course more and better observational data coming from the already discovered habitable SEs and future discovered potentially habitable exoplanets will allow us to assess the actual magnetic protection of potentially habitable environments.

We appreciate the useful discussion and comments of Mercedes Lopez-Morales and other colleagues participating in the Exploring Strange New Worlds 2011 Conference (Arizona, U.S.) and in the VI Taller de Ciencias Planetarias 2012 (Montevideo, Uruguay). Special thanks to Ignacio Ferrin who, with his clever questions and suggestions, originally motivated us to pursue some of the goals of this work. We also thank Lisa Kaltenegger and Jeffrey Linsky for their insightful comments on preliminary versions of this manuscript. We give special thanks to all our fellow colleagues abroad who provided us with some key pieces of literature unobtainable in our country. We are grateful to Aaron West and Luke Webb for their careful revision of the English in the manuscript. The remnant errors are all ours. An anonymous referee is acknowledged for contributing significantly not only to the improvement of the manuscript but also to the quality of the research conclusions. P.C. is supported by the Vicerrectoria de Docencia of the University of Antioquia. This work has been completed with the financial support of the CODI-UdeA under the grant IN591CE and by the University of Medellin under the grant number 530 .

\section{REFERENCES}

Aubert, J., Labrosse, S., \& Poitou, C. 2009, GeoJI, 179, 1414

Baraffe, I., Chabrier, G., Allard, F., \& Hauschildt, P. H. 1998, A\&A, 337, 403

Bastian, T. S., Dulk, G. A., \& Leblanc, Y. 2000, ApJ, 545, 1058

Batalha, N. M., Rowe, J. F., Bryson, S. T., et al. 2012, ApJS, 204, 24

Bonfils, X., Delfosse, X., Udry, S., et al. 2011, arXiv:1111.5019B

Borucki, W. J., Koch, D. G., Basri, G., et al. 2011, ApJ, 728, 117

Boss, A. P. 2006, ApJL, 644, L79

Catanzarite, J., \& Shao, M. 2011, ApJ, 738, 151

Chaufray, J. Y., Modolo, R., Leblanc, F., et al. 2007, JGRE, 112, 9009

Christensen, U., Balogh, A., Breuer, D., \& Glaßmeier, K. 2009, Planetary

Magnetism, Space Sciences Series of ISSI (New York: Springer)

Christensen, U. R. 1985, JGR, 90, 2995

Christensen, U. R. 2010, SSRv, 152, 565

Cohen, O., Kashyap, V. L., Drake, J. J., Sokolov, I. V., \& Gombosi, T. I. 2011, ApJ, 738, 166

Denis, C., Rybicki, K. R., Schreider, A. A., Tomecka-Suchoń, S., \& Varga, P. 2011, AN, 332, 24

Driscoll, P., \& Olson, P. 2011, Icar, 213, 12

Elkins-Tanton, L. T., \& Seager, S. 2008, ApJ, 688, 628

Farrell, W. M., Lazio, T. J. W., Desch, M. D., Bastian, T. S., \& Zarka, P. 2004, in IAU Symp. 213, Bioastronomy 2002: Life Among the Stars, ed. R. Norris \& F. Stootman (San Francisco, CA: ASP), 73

Ferraz-Mello, S., Rodríguez, A., \& Hussmann, H. 2008, CeMDA, 101, 171

Gaidos, E., Conrad, C. P., Manga, M., \& Hernlund, J. 2010, ApJ, 718, 596

Garcés, A., Catalán, S., \& Ribas, I. 2011, A\&A, 531, A7

Grenfell, J. L., Stracke, B., von Paris, P., et al. 2007, P\&SS, 55, 661

Grießmeier, J.-M., Khodachenko, M., Lammer, H., et al. 2010, in IAU Symp. 264, Solar and Stellar Variability: Impact on Earth and Planets, ed. A. G. Kosovichev, A. H. Andrei, \& J.-P. Rozelot (Cambridge: Cambridge Univ. Press), 385

Grießmeier, J.-M., Preusse, S., Khodachenko, M., et al. 2007a, P\&SS, 55, 618

Grießmeier, J.-M., Stadelmann, A., Grenfell, J. L., Lammer, H., \& Motschmann, U. 2009, Icar, 199, 526

Grießmeier, J.-M., Stadelmann, A., Motschmann, U., et al. 2005, AsBio, 5, 587

Grießmeier, J.-M., Stadelmann, A., Penz, T., et al. 2004, A\&A, 425, 753

Grießmeier, J.-M., Zarka, P., \& Spreeuw, H. 2007b, A\&A, 475, 359

Gubbins, D., Alfè, D., Masters, G., Price, G. D., \& Gillan, M. 2004, GeoJI, 157,1407

Gubbins, D., Alfè, D., Masters, G., Price, G. D., \& Gillan, M. J. 2003, GeoJI, 155,609

Guinan, E. F., Engle, S. G., \& Dewarf, L. E. 2009, in AIP Conf. Ser. 1135, ed M. E. van Steenberg, G. Sonneborn, H. W. Moos, \& W. P. Blair (Melville, NY: AIP), 244

Heller, R., Barnes, R., \& Leconte, J. 2011, OLEB, 41, 539

Henning, W. G., O’Connell, R. J., \& Sasselov, D. D. 2009, ApJ, 707, 1000

Joshi, M. M., Haberle, R. M., \& Reynolds, R. T. 1997, Icar, 129, 450

Judge, P. G., Solomon, S. C., \& Ayres, T. R. 2003, ApJ, 593, 534

Kaltenegger, L. 2010, ApJL, 712, L125

Kaltenegger, L., Udry, S., \& Pepe, F. 2011, arXiv:1108.3561

Kasting, J. 2010, How to Find a Habitable Planet (Princeton, NJ: Princeton Univ. Press)

Kasting, J. F., Whitmire, D. P., \& Reynolds, R. T. 1993, Icar, 101, 108

Khodachenko, M. L., Ribas, I., Lammer, H., et al. 2007, AsBio, 7, 167

Kipping, D. M., Bakos, G. Á., Buchhave, L., Nesvorný, D., \& Schmitt, A. 2012, ApJ, 750, 115

Kite, E. S., Gaidos, E., \& Manga, M. 2011, ApJ, 743, 41

Kite, E. S., Manga, M., \& Gaidos, E. 2009, ApJ, 700, 1732

Kopparapu, R. K., Ramirez, R., Kasting, J. F., et al. 2013, ApJ, 765, 131

Kulikov, Y. N., Lammer, H., Lichtenegger, H. I. M., et al. 2006, P\&SS, 54, 1425

Labrosse, S. 2003, PEPI, 140, 127

Labrosse, S., Poirier, J.-P., \& Le Mouël, J.-L. 2001, E\&PSL, 190, 111

Lammer, H., Bredehöft, J. H., Coustenis, A., et al. 2009, A\&ARv, 17, 181

Lammer, H., Lichtenegger, H. I. M., Khodachenko, M. L., Kulikov, Y. N., \&

Griessmeier, J. 2012, in ASP Conf. Ser. 450, The Loss of Nitrogen-rich

Atmospheres from Earth-Like Exoplanets within M-star Habitable Zones,

ed. J. P. Beaulieu, S. Dieters, \& G. Tinetti (San Francisco, CA: ASP), 139

Lammer, H., Lichtenegger, H. I. M., Kulikov, Y. N., et al. 2007, AsBio, 7, 185

Lammer, H., Ribas, I., Grießmeier, J.-M., et al. 2004, HvaOB, 28, 139 
Lammer, H., Selsis, F., Chassefière, E., et al. 2010, AsBio, 10, 45

Lammer, H., Selsis, F., Ribas, I., et al. 2003, ApJL, 598, L121

Lazio, J., Bastian, T., Bryden, G., et al. 2009, Astronomy, Vol. 2010, Astro2010: The Astronomy and Astrophysics Decadal Survey, Science White Papers, No. 177

Leconte, J., Chabrier, G., Baraffe, I., \& Levrard, B. 2010, A\&A, 516, A64

López-Morales, M., Gómez-Pérez, N., \& Ruedas, T. 2011, OLEB, 41, 533

Manga, M., Weeraratne, D., \& Morris, S. J. S. 2001, PhFl, 13, 802

Mayor, M., Bonfils, X., Forveille, T., et al. 2009, A\&A, 507, 487

Mayor, M., \& Udry, S. 2008, PhST, 130, 014010

Mead, G. D. 1964, JGR, 69, 1181

Miguel, Y., \& Brunini, A. 2010, MNRAS, 406, 1935

Neudegg, D. A., Cowley, S. W. H., McWilliams, K. A., et al. 2001, AnGeo, 19,179

Newkirk, G., Jr. 1980, in The Ancient Sun: Fossil Record in the Earth, Moon and Meteorites, ed. R. O. Pepin, J. A. Eddy, \& R. B. Merrill (New York: Pergamon), 293

Nimmo, F. 2009, Treatise on Geophysics, Vol. 8 (Amsterdam: Elsevier), 31

Nimmo, F., \& Stevenson, D. J. 2000, JGR, 105, 11969

Olson, P., \& Christensen, U. R. 2006, E\&PSL, 250, 561

Papuc, A. M., \& Davies, G. F. 2008, Icar, 195, 447

Parker, E. N. 1958, ApJ, 128, 664

Peale, S. J. 1977, in IAU Colloq. 28: Planetary Satellites, ed. J. A. Burns (Tucson, AZ: Univ. Arizona Press), 87

Pepe, F., Lovis, C., Ségransan, D., et al. 2011, A\&A, 534, A58

Pozzo, M., Davies, C., Gubbins, D., \& Alfè, D. 2012, Natur, 485, 355

Preusse, S., Kopp, A., Büchner, J., \& Motschmann, U. 2005, A\&A, 434, 1191

Rauer, H., Gebauer, S., Paris, P. V., et al. 2011, A\&A, 529, A8

Ricard, Y. 2009, Treatise on Geophysics, Vol. 7 (Amsterdam: Elsevier), 6054

Scalo, J., Kaltenegger, L., Segura, A. G., et al. 2007, AsBio, 7, 85

Schubert, G., Cassen, P., \& Young, R. E. 1979, Icar, 38, 192

Schubert, G., Turcotte, D. L., \& Olson, P. 2001, Mantle Convection in the Earth and Planets (Cambridge: Cambridge Univ. Press)

Schwenn, R. 1990, Large-Scale Structure of the Interplanetary Medium (Berlin: Springer), 99

Segura, A., Walkowicz, L. M., Meadows, V., Kasting, J., \& Hawley, S. 2010, AsBio, 10, 751
Selsis, F., Kasting, J. F., Levrard, B., et al. 2007, A\&A, 476, 1373

Siscoe, G., \& Christopher, L. 1975, GeoRL, 2, 158

Siscoe, G., \& Crooker, N. 1976, JGG, 28, 1

Siscoe, G. L., \& Chen, C.-K. 1975, JGR, 80, 4675

Stacey, F. D. 1992, Physics of the Earth (Brisbane: Brookfield Press)

Stamenković, V., Breuer, D., \& Spohn, T. 2011, Icar, 216, 572

Stamenković, V., Noack, L., Breuer, D., \& Spohn, T. 2012, ApJ, 748,41

Stevenson, D. J. 1983, RPPh, 46, 555

Stevenson, D. J. 2003, E\&PSL, 208, 1

Stevenson, D. J. 2010, SSRv, 152, 651

Tachinami, C., Senshu, H., \& Ida, S. 2011, ApJ, 726, 70

Tarduno, J. A., Cottrell, R. D., Watkeys, M. K., et al. 2010, Sci, 327, 1238

Tian, F. 2009, ApJ, 703, 905

Tian, F., Kasting, J. F., Liu, H.-L., \& Roble, R. G. 2008, JGRE, 113, 5008

Tuomi, M., Anglada-Escudé, G., Gerlach, E., et al. 2012, A\&A, 549, A48

Udry, S., Bonfils, X., Delfosse, X., et al. 2007, A\&A, 469, L43

Underwood, D. R., Jones, B. W., \& Sleep, P. N. 2003, IJAsB, 2, 289

Valencia, D., O'Connell, R. J., \& Sasselov, D. 2006, Icar, 181, 545

Valencia, D., Sasselov, D. D., \& O’Connell, R. J. 2007, ApJ, 665, 1413

Varga, P., Denis, C., \& Varga, T. 1998, JGeod, 25, 61

Vidotto, A. A., Jardine, M., \& Helling, C. 2010, ApJL, 722, L168

Vidotto, A. A., Jardine, M., \& Helling, C. 2011, MNRAS, 411, L46

Voigt, G. 1995, Handbook of Atmospheric Electrodynamics, Vol. 2 (Boca Raton, FL: CRC Press), 333

Ward, P., \& Brownlee, D. 2000, Rare Earth: Why Complex Life is Uncommon in the Universe (New Yokr: Copernicus Books)

Watson, A. J., Donahue, T. M., \& Walker, J. C. G. 1981, Icar, 48, 150

Weber, E. J., \& Davis, L., Jr. 1967, ApJ, 148, 217

Weinstein, S. A. 1992, E\&PSL, 113, 23

Wood, B. E., Müller, H.-R., Zank, G. P., \& Linsky, J. L. 2002, ApJ, 574,412

Wood, B. E., Müller, H.-R., Zank, G. P., Linsky, J. L., \& Redfield, S. 2005, ApJL, $628, \mathrm{~L} 143$

Yamazaki, D., \& Karato, S. 2001, AmMin, 86, 385

Zendejas, J., Segura, A., \& Raga, A. C. 2010, Icar, 210, 539

Zuluaga, J. I., \& Cuartas, P. A. 2012, Icar, 217, 88 